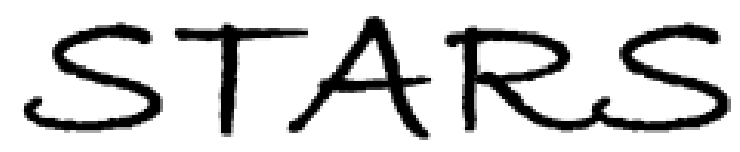

University of Central Florida

STARS

$1-1-1998$

\title{
Exponential asymptotic expansions and approximations of the unstable and stable manifolds of singularly perturbed systems with the Henon map as an example
}

\author{
Alexander Tovbis \\ University of Central Florida \\ Masa Tsuchiya \\ Charles Jaffé
}

Find similar works at: https://stars.library.ucf.edu/facultybib1990

University of Central Florida Libraries http://library.ucf.edu

This Article is brought to you for free and open access by the Faculty Bibliography at STARS. It has been accepted for inclusion in Faculty Bibliography 1990s by an authorized administrator of STARS. For more information, please contactSTARS@ucf.edu.

\section{Recommended Citation}

Tovbis, Alexander; Tsuchiya, Masa; and Jaffé, Charles, "Exponential asymptotic expansions and approximations of the unstable and stable manifolds of singularly perturbed systems with the Henon map as an example" (1998). Faculty Bibliography 1990s. 2479.

https://stars.library.ucf.edu/facultybib1990/2479

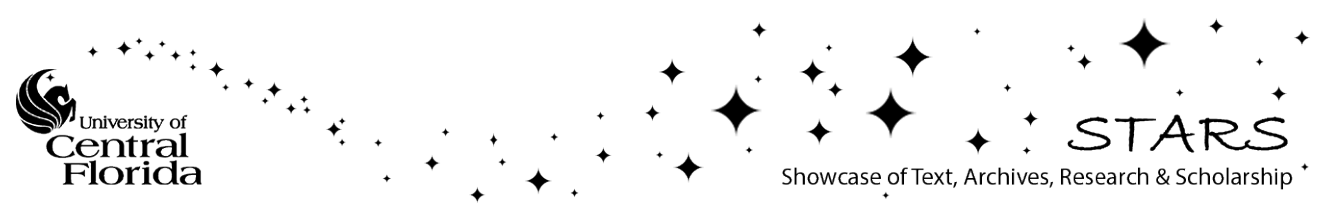




\section{Exponential asymptotic expansions and approximations of the unstable and stable manifolds of singularly perturbed systems with the Hénon map as an example}

Cite as: Chaos 8, 665 (1998); https://doi.org/10.1063/1.166349

Submitted: 12 May 1997. Accepted: 09 February 1998 . Published Online: 31 August 1998

Alexander Tovbis, Masa Tsuchiya, and Charles Jaffé

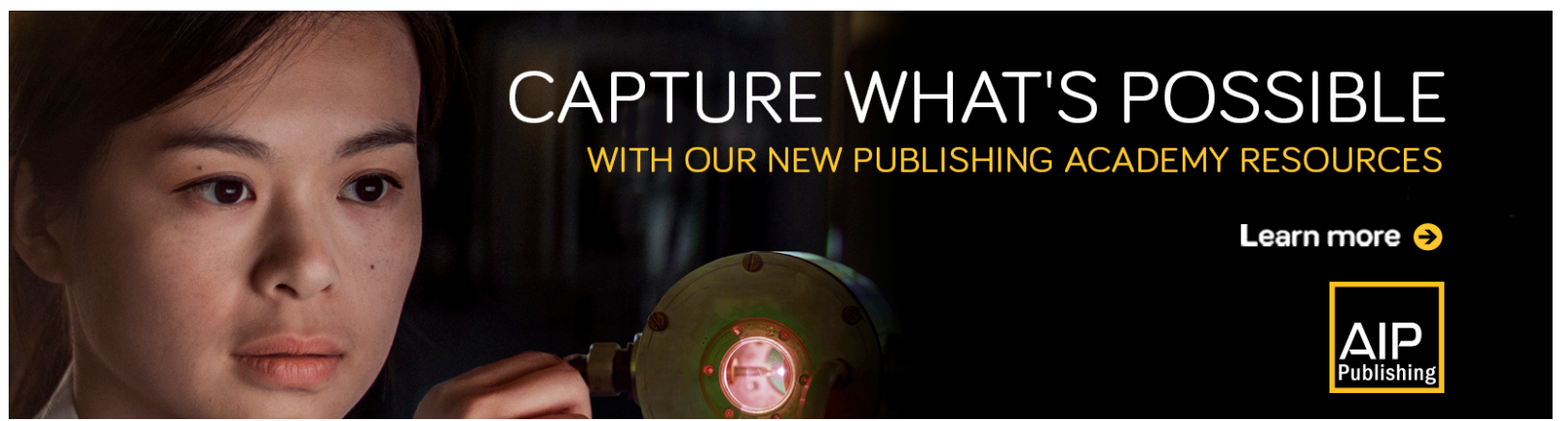




\title{
Exponential asymptotic expansions and approximations of the unstable and stable manifolds of singularly perturbed systems with the Hénon map as an example
}

\author{
Alexander Tovbis ${ }^{\text {a) }}$ \\ Department of Mathematics, University of Central Florida, Orlando, Florida 32816-1364 \\ Masa Tsuchiya ${ }^{\text {b) }}$ \\ Department of Physics, West Virginia University, Morgantown, West Virginia 26506-6315 \\ Charles Jafféc) \\ Department of Chemistry, West Virginia University, Morgantown, West Virginia 26506-6045
}

(Received 12 May 1997; accepted for publication 9 February 1998)

\begin{abstract}
The subject of this paper is the construction of the exponential asymptotic expansions of the unstable and stable manifolds of the area-preserving Hénon map. The approach that is taken enables one to capture the exponentially small effects that result from what is known as the Stokes phenomenon in the analytic theory of equations with irregular singular points. The exponential asymptotic expansions were then used to obtain explicit functional approximations for the stable and unstable manifolds. These approximations are compared with numerical simulations and the agreement is excellent. Several of the main results of the paper have been previously announced in A. Tovbis, M. Tsuchiya, and C. Jaffé ["Chaos-integrability transition in nonlinear dynamical systems: exponential asymptotic approach," Differential Equations and Applications to Biology and to Industry, edited by M. Martelli, K. Cooke, E. Cumberbatch, B. Tang, and H. Thieme (World Scientific, Singapore, 1996), pp. 495-507, and A. Tovbis, M. Tsuchiya, and C. Jaffé, "Exponential asymptotic expansions and approximations of the unstable and stable manifolds of the Hénon map," preprint, 1994]. (C) 1998 American Institute of Physics. [S1054-1500(98)00602-8]
\end{abstract}

It is well known that perturbed integrable nonlinear systems may give rise to chaotic dynamics. It seems natural therefore to apply perturbation methods to study the "onset of chaos" in such systems. The difficulty, however, is that in certain situations the effect of the perturbation is exponentially small with respect to the perturbation parameter. Thus some of the recently developed methods of exponential asymptotics are needed. In this paper these methods are used and further developed in order to obtain a closed analytical form approximation to the stable and unstable manifolds. The area-preserving Hénon map, or equivalently, the discretized equation $y^{\prime \prime}=y^{2}-2 y$, is considered as a model example. Comparisons of our approximation with numerical results for the manifolds and for the homoclinic tangle are presented.

\section{INTRODUCTION}

We focus our attention on the onset of chaotic behavior in singularly perturbed systems caused by splitting of separatrices. In particular, we are interested in obtaining analytic

\footnotetext{
${ }^{\text {a)} E l e c t r o n i c ~ m a i l: ~ a t o v b i s @ ~ @ e g a s u s . c c . u c f . e d u ~}$

${ }^{b}$ Current address: Department of Chemistry, Cornell University, Ithaca, NY 14853. Electronic mail: mt58@cornell.edu

${ }^{c)}$ Electronic mail: cjaffe@wvu.edu
}

expressions to approximate the stable and unstable manifolds in such perturbed systems for long time intervals. It is known that generically such perturbations cause exponentially small splittings with respect to the small parameter (the "difficult" case in the terminology of Ref. 1, see the introduction) and therefore the standard Melnikov's technique is not applicable here.

The case of exponentially small separatrix splitting, as it was first noticed by Poincaré, is extremely important for physical applications. It was first observed for rapidly forced systems (see, for example, Ref. 2), and then for singularly perturbed and discretized systems. ${ }^{3,4}$ It is known that a constant step discretization of a system can be viewed as a rapid forcing of this system. ${ }^{5}$ In this paper we restrict our attention on the dynamics of singularly perturbed systems.

As a demonstration of physical importance of such systems, we can mention, for example, that the onset of chaotic motion in a unimolecular reaction (a system of coupled linear and quadratic oscillators) could be considered through the mechanism studied below. Another example that demonstrates the importance of the mechanism is saddle-center bifurcations of the Hénon map. This map is often used to model Poincaré surfaces of section for two degree of freedom Hamiltonian systems in the natural sciences. ${ }^{6}$ Approximation of the splitting separatrices for long time intervals allows to compute the so-called "lobe" areas (see, for ex- 
ample, Ref. 7), and thus to obtain quantities which characterize reaction rates in a variety of chemical problems.

The approach that we take is to apply the technique of exponential asymptotic expansions (also known as asymptotics beyond all orders, see Ref. 8) based on Ref. 3. The strength of this approach lies in its ability to capture the effect of terms that are exponentially small in the perturbation parameter. These terms, however, become critical in the long time limit.

Originally, Kruskal and Segur ${ }^{3}$ developed their method in order to show that a simple geometrical model of crystal growth does not possess needle crystal solutions. That is, the solution

$$
v(t)=-\frac{\pi}{2}+2 \tan ^{-1}\left(e^{t}\right)
$$

to the equation

$$
v^{\prime}=\cos v
$$

that has asymptotics $v(t) \sim \pm \pi / 2$ as $t \rightarrow \pm \infty$, does not survive the singular perturbation

$$
\varepsilon^{2} v^{\prime \prime \prime}+v^{\prime}=\cos v
$$

Or, in other words, the perturbed equation (3) does not possess a heteroclinic solution.

To establish this fact, the following strategy was suggested: construct two solutions $v_{s}(t)$ and $v_{u}(t)$ to (3), that approach $\pm \pi / 2$ as $t \rightarrow \pm \infty$, and then compare these solutions at some finite point $t_{0} \in \mathbf{C}$. This point is taken at one of the two poles of (1) that are closest to the real $t$ axis; say $t_{0}$ $=i \pi / 2$. One can view this choice of $t_{0}$ as motivated as follows: the difference $v_{s}(t)-v_{u}(t)$ is known to be exponentially small in $\varepsilon$; however, the exponentially small factor can be compensated by some growing factor as $t \rightarrow i \pi / 2$, so that $v_{s}(t)-v_{u}(t)$ can become detectable in a proper scaling of the variables.

This scaling is given in Ref. 3 by

$$
\begin{aligned}
& t=\frac{i \pi}{2}+\varepsilon z, \\
& v(t, \varepsilon)=-\frac{\pi}{2}+i \ln \frac{2}{\varepsilon}+i \Phi(z, \varepsilon) .
\end{aligned}
$$

It reduces the perturbed equation (3) (called henceforth outer equation) to

$$
\Phi^{\prime \prime \prime}+\Phi^{\prime}=e^{\Phi}-\left(\frac{\varepsilon}{2}\right)^{2} e^{-\Phi}
$$

(called the inner equation). The advantage of (5) versus (3) is that the difference between stable and unstable solutions can be detected in the leading-order terms equation

$$
\Phi^{\prime \prime \prime}+\Phi^{\prime}=e^{\Phi}
$$

of (5), which is referred to as truncated inner equation. At the same time, as we have mentioned, the difference $v_{s}(t)$ $-v_{u}(t)$ is exponentially small in $\varepsilon$ and therefore cannot be captured in any order of $\varepsilon$ expansion of (3), see Sec. II. This makes $v_{s}(t)-v_{u}(t)$ "'beyond all orders.'

Equation (6) possesses the formal power series solution

$$
\hat{\Phi}=-\ln (-z)+\sum_{k=1}^{\infty} \frac{a_{k}}{z^{2 k}}, \quad a_{k} \in \mathbf{R} .
$$

This implies that there are actual solutions of (6) possessing asymptotic expansion (7). Moreover (see, for example, Ref. 9 ), these actual solutions $\Phi_{ \pm}(z)$ to (6) are uniquely defined by

$$
\Phi_{ \pm}(z) \sim \hat{\Phi}(z), \quad z \rightarrow \infty, \quad \pm \Re z>0 .
$$

In Ref. 3 the solutions $\Phi_{ \pm}(z)$ were put into correspondence with the stable and unstable solutions $v_{s}(t)$ and $v_{u}(t)$ of (3), respectively. Some nonrigorous analytical methods, together with numerical arguments, were employed to show that $\Phi_{+}(z) \not \equiv \Phi_{-}(z)$ and, as a consequence, $v_{s}(t) \not \equiv v_{u}(t)$. So, the perturbed equation (3) does not have needle crystal solutions. (A rigorous proof of this fact using different techniques can be found in Ref. 10; another interesting treatment, though less rigorous, can be found in Ref. 11. The rigorous treatment of a similar singularly perturbed fourth-order equation can be found in Refs. 12 and 13.)

It was noticed in Ref. 9 that the fact that $\Phi_{+}(z)$ does not coincide with $\Phi_{-}(z)$ can be proved rigorously: it follows from the observation that Eq. (6) has nontrivial Stokes phenomenon at $z=\infty$. The consequent observation is that more detailed study of the Stokes phenomenon [i.e., study of behavior of $\Phi_{ \pm}(z)$ in a neighborhood of $z=\infty$ ] can provide more information about the stable and unstable solutions $v_{s}(t)$ and $v_{u}(t)$ of (3).

Since the appearance of Ref. 3 as a preprint in 1985, the "asymptotic beyond all orders" technique was applied, mainly on the formal level, to show nonexistence of homoclinic or heteroclinic solutions to singularly perturbed ODEs, which correspond to various singularly perturbed problems: shock waves to the perturbed KuramotoSivashinsky equation, ${ }^{14}$ traveling waves to the fifth-order Korteweg-de Vries (KdV) equation, ${ }^{15,16}$ some discretized problems, ${ }^{17,18}$ etc. In these papers the study of the truncated inner equations (as in Ref. 3) was either done numerically or by the Borel summation, formally applied to the corresponding formal power series solutions of type (7). The latter allows to compute the Stokes constant $c$ (see Ref. 19 for the definition of $c$ ). The fact that $c \neq 0$ means that the Stokes phenomenon is nontrivial. The formal use of the Borel summation for the above-mentioned equations was justified in Ref. 9. As it was also mentioned there, the mere fact that the formal power series solutions to the truncated inner equation are divergent (have zero radius of convergence) in certain cases means that the corresponding Stokes phenomena are nontrivial. So, the computation of $c$ is not needed to show just the nonexistence of the corresponding heteroclinic or homoclinic solution. However, it was noticed that the splitting quantities [such as the distance between $v_{s}(t)$ and $v_{u}(t)$, angles of intersection of the corresponding phase curves, projected on the $\left(v, v^{\prime}\right)$ plane] are proportional to $c$. So, approximations of these exponentially small quantities near the value $t=0$ [near the first point of intersection of the corresponding curves on the $\left(v, v^{\prime}\right)$ plane] could be obtained (see, for example, Refs. 14, 16, and 20) by computing $c$. 
It is well known that a difference operator can be considered as an infinite order differential operator. Accordingly, a finite difference approximation to an ODE can be considered as a singular perturbation, where the step size of the discretization plays the role of a small parameter. A discretized pendulum equation, which is equivalent to the standard Chirikov's map, was considered in Ref. 17. The authors formally applied the formal method of Ref. 3 to show splitting of the separatrix and to obtain the asymptotic result of Lazutkin et $a{ }^{21}{ }^{21}$ for the splitting angle. It is worth mentioning that the main technique used by Lazutkin (later joined by his colleagues) to study the standard map, is in fact parallel to that of Ref. 3 and was developed at approximately the same time.

The starting point of our arguments is that we can obtain more information about $v_{s}(t)$ and $v_{u}(t)$ by more detailed study of the corresponding solutions $\Phi_{ \pm}(z)$ to the truncated inner equations. In particular, we can express the difference $\Phi_{+}(z)-\Phi_{-}(z)$ as a convergent series of exponentially small corrections. This enables us to construct a double expansion in powers of $\varepsilon$ and $e^{-\alpha / \varepsilon}$, with some $\alpha>0$. This expansion formally satisfies the original perturbed equation. Based on the above-mentioned expansion, we derive an analytic expression to approximate $v_{s}(t)$ and $v_{u}(t)$ on sufficiently long $t$ intervals, which is called a first-order approximation.

As a model example of a singularly perturbed problem we consider the central finite difference approximation:

$$
\frac{y(t+\sigma)-2 y(t)+y(t-\sigma)}{\sigma^{2}}=y^{2}(t)-2 y(t)
$$

of a particle in the cubic potential:

$$
y^{\prime \prime}(t)=y^{2}(t)-2 y(t) \text {. }
$$

In Sec. II we discuss the equivalence of (9) to the area preserving Hénon map, give simple arguments proving the splitting of the separatrix solution of (10) under the perturbation (9), show that the difference between the stable $y_{s}(t, \sigma)$ and unstable $y_{u}(t, \sigma)$ solutions to (9) is beyond all orders of $O\left(\sigma^{n}\right)$, rescale Eq. (9) according to Ref. 3 and determine solutions $\Phi_{s}(t)$ and $\Phi_{u}(t)$ of the truncated inner equation that correspond to $y_{s}$ and $y_{u}$. Much of the material in this section and the following section reviews material essential for the following discussion. Related material for particular maps (for example, for the standard map), as well as for certain classes of analytic maps can be found in a number of publications (see, for example, Refs. 21-24).

In the third section we define irregular singularities and discuss the Stokes phenomenon at an irregular singular point on one of the simplest examples. We then study the Stokes phenomenon for the truncated inner equation by the means of the Borel-Laplace method. Exponential expansion satisfying the equation is derived next, and the Stokes constant $c$ is evaluated. Based on the relation between the original perturbed equation (9) and the truncated inner equation, we first derive the double expansion satisfying (9), and then obtain the first-order approximation of the unstable manifold:

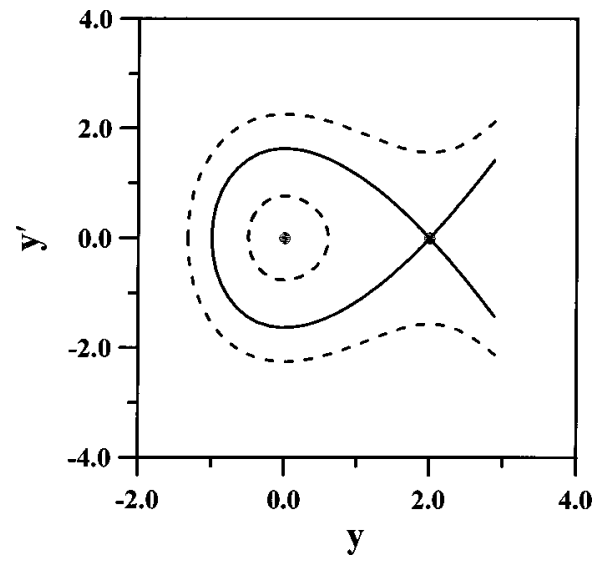

FIG. 1. The phase plane $\left(y, y^{\prime}\right)$ of the unperturbed equation (11). The curves of constant $\Psi$ define phase trajectories $\left(y(t), y^{\prime}(t)\right)$, while the other constant $\psi$ defines the origin of time $t$.

$$
\begin{aligned}
y(t, \sigma)= & y_{00}(t)+\sigma^{2} y_{01}(t)+\frac{84 \pi K}{\sigma^{6}} e^{-\sqrt{2} \pi^{2} / \sigma} \\
& \times S(t)\left[v_{2}(t) \sin \frac{2 \pi t}{\sigma}-\frac{5}{48 \sqrt{2}} v_{1}(t) \cos \frac{2 \pi t}{\sigma}\right]
\end{aligned}
$$

that takes into account exponentially small terms of the leading order. The functions $y_{00}, y_{01}, v_{1}$, and $v_{2}$ are expressed via hyperbolic functions in closed form; $2 \pi i K=c$ is the Stokes constant, and the function $S(t)$ monotonically increases from 0 to 2 as $t$ is varied from $-\infty$ to $+\infty$. These functions are discussed in Sec. IV where one can also find the discussion of our approximation and its compatibility with numerical simulations (which, overall, is excellent).

The preliminary version of this paper was presented at the International Conference on Differential Equations and Applications to Biology and Industry, Claremont, CA, 1994. It appeared as a preprint also in $1994 .^{25} \mathrm{~A}$ short version (preliminary research report) was published in the Proceedings of the Conference. ${ }^{26}$ Since then the suggested technique (below) was applied to study a double-well potential case in Refs. 27 and 28.

\section{THE PERTURBED SYSTEM IN THE ORIGINAL AND SCALED VARIABLES}

\section{A. The perturbed equation}

Consider the second-order differential equation

$$
y^{\prime \prime}(t)=y^{2}(t)-2 y(t) .
$$

Following standard procedures, one obtains the general solution of (11) as

$$
y(t)=1+6 \mathfrak{p}(t+\psi),
$$

where $\mathfrak{p}$ is the Weierstrass elliptic function ${ }^{29}$ with the invariants $g_{2}=1 / 3, g_{3}=1 / 27-\Psi$. Here $\Psi$ and $\psi$ are arbitrary complex constants. One can interpret (11) as the equation of a particle in the cubic potential (one-well potential) field

$$
V(y)=+y^{2}-\frac{1}{3} y^{3} .
$$


with total energy $E=18 \Psi$. The value of $\Psi$ determines whether the solutions of (11) are bounded or unbounded. In the phase plane $\left(y, y^{\prime}\right)$ of (11) (see Fig. 1), curves of constant $\Psi$ define phase trajectories $\left[y(t), y^{\prime}(t)\right]$, while the other constant $\psi$ defines the origin of the time $t$. Equation (11) possesses an elliptic fixed point $(0,0)$ and a hyperbolic fixed point $(2,0)$ in the phase plane. The special values of the parameters $\Psi=2 / 17$ and $\psi=i \pi / \sqrt{2}$ correspond to the solution

$$
y(t)=-1+3 \tanh ^{2}\left(\frac{t}{\sqrt{2}}\right) .
$$

This solution defines the separatrix in the phase plane that is the boundary between bounded and unbounded phase trajectories. The separatrix is a homoclinic trajectory that asymptotically approaches the hyperbolic point $(2,0)$ in the future $(t \rightarrow \infty)$ and in the past $(t \rightarrow-\infty)$. We will refer to the solution (14) as to the separatrix solution. The hyperbolic fixed point $(2,0)$ possesses stable and unstable manifolds (incoming and outgoing trajectories). These are the phase trajectories of a particle reaching or escaping this fixed point. For Eq. (11), both stable and unstable manifolds join smoothly to form the separatrix.

Our aim is to study the stable and unstable manifolds (and the corresponding solutions) of the perturbed equation

$$
\frac{y(t+\sigma)-2 y(t)+y(t-\sigma)}{\sigma^{2}}=y^{2}(t)-2 y(t),
$$

where $\sigma$ is a small positive parameter. By a solution of (15) we mean a function $y(t)$ of real (or of complex) variable $t$ satisfying (15). Eq. (15) is a finite difference approximation (discretization) of the unperturbed equation (11). We refer to (15) as to the perturbed equation.

\section{B. The Hénon map}

Eq. (15) arises naturally from the rescaling of the areapreserving Hénon map ${ }^{30}$

$$
\begin{aligned}
& u_{i+1}=-v_{i}+\beta u_{i}-\lambda u_{i}^{2}, \\
& v_{i+1}=u_{i} .
\end{aligned}
$$

Here $\beta=2\left(1-\sigma^{2}\right), \lambda=\sigma^{4}-1$, and we are interested in the limiting behavior as $\sigma \rightarrow 0$. In this limit the fixed points $(u, v)=(0,0)$ and $(u, v)=\left(2 \sigma^{2} /\left(1-\sigma^{4}\right), 2 \sigma^{2} /\left(1-\sigma^{4}\right)\right)$ collide. This leads to difficulties in examining the limiting behavior as $\sigma \rightarrow 0$. In order to avoid these difficulties we introduce new scaled variables

$$
u_{i}=\frac{\sigma^{2}}{1-\sigma^{4}} y_{i}
$$

The corresponding equation for $y_{i}$ is

$$
\frac{y_{i+1}-2 y_{i}+y_{i-1}}{\sigma^{2}}=y_{i}^{2}-2 y_{i} \text {. }
$$

The second-order difference equation (18) is in fact the Euler's finite-difference scheme with the step $\sigma$ for (11).
Substituting $y_{i}=y(t), y_{i \pm 1}=y(t \pm \sigma)$, we obtain (15). Thus the unperturbed equation (11) can be considered as the blowup limit of (16) when $\sigma \rightarrow 0$.

\section{Discretization is a singular perturbation}

It is well known that a difference equation can be considered as an ordinary differential equation of infinite order (see, for example, Ref. 31). In order to put (15) in this form, we use the Taylor series representation

$$
y(t \pm \sigma)=y(t) \pm \sigma y^{\prime}(t)+\sum_{k=2}^{\infty}( \pm 1)^{k} \frac{\sigma^{k}}{k !} y^{(k)}(t) .
$$

Then, the symmetrical second difference is

$$
\begin{aligned}
\Delta_{\sigma}^{2} y & =\frac{y(t+\sigma)-2 y(t)+y(t-\sigma)}{\sigma^{2}} \\
& =2 \sum_{j=1}^{\infty} \frac{\sigma^{2(j-1)}}{(2 j) !} y^{(2 j)}(t),
\end{aligned}
$$

and (15) becomes

$$
y^{\prime \prime}(t)=y^{2}(t)-2 y(t)-2 \sum_{j=2}^{\infty} \frac{\sigma^{2(j-1)}}{(2 j) !} y^{(2 j)}(t) .
$$

Equation (21) is an infinite-order differential equation and simultaneously is a singular perturbation of the original equation (11) (the perturbation term contains higher derivatives multiplied by powers of the small parameter).

The study of the Hénon map is not the only situation when a singular perturbation of (11) is of interest. For example, another singular perturbation

$$
y^{\prime \prime}(t)=y^{2}(t)-2 y(t)-\sigma^{2} y^{\prime \prime \prime}(t)
$$

occurs in the study of traveling wave solutions to the perturbed Korteweg-de Vries equation (see Refs. 15 and 16).

\section{The stable and unstable manifolds and the splitting of the separatrix}

We are interested in the consequences of the singular perturbation for the separatrix solution of the unperturbed equation. The following arguments show that this solution does not survive the perturbation. Thus (15) does not have a separatrix solution.

The difference equation (15) defines the diffeomorphism $T: \mathbf{R}^{2} \rightarrow \mathbf{R}^{2}$ which is [ equivalent to the Hénon map (16)] by

$$
\begin{aligned}
& \eta_{i+1}=\eta_{i}+\sigma\left(\xi_{i}+\sigma\left(\eta_{i}^{2}-2 \eta_{i}\right)\right), \\
& \xi_{i+1}=\xi_{i}+\sigma\left(\eta_{i}^{2}-2 \eta_{i}\right),
\end{aligned}
$$

where $\quad \eta=y(t), \quad \xi=\Delta_{\sigma} y(t) \equiv[y(t)-y(t-\sigma)] / \sigma$. The point $(2,0)$ is a hyperbolic fixed point of (23). Associated with this fixed point there exist two one-dimensional manifolds: the stable $W_{s}$ and the unstable $W_{u}$ manifolds (see, for example, Ref. 32, Chap. 2).

A solution $y(t, \sigma)$ of $(15)$ that satisfies

$$
\lim _{t \rightarrow+\infty} y_{s}(t, \sigma)=2, \quad y_{s}(t, \sigma)<2 \quad \text { as } t \rightarrow+\infty
$$


is called a stable solution. Since $W_{s}$ is one dimensional it has to coincide with the graph of $\left(y_{s}(t), \Delta_{\sigma} y_{s}(t)\right)$. This solution $y_{s}$ is invariant with respect to the shifting of the origin of time, that is, if $y_{s}(t)$ is a stable solution then $y_{s}\left(t+t_{0}\right)$, where $t_{0} \in \mathbf{R}$, is also a stable solution. A solution satisfying (24) as $t \rightarrow-\infty$ is called an unstable solution.

The fact that $W_{s} \neq W_{u}$ was proven in Ref. 33. Presented below are different arguments that lead to the same conclusion. According to the Poincare theorem (see, for example, Ref. 34), a stable solution $y_{s}(t, \sigma)$ can be represented by the series

$$
y_{s}(t, \sigma)=2+\sum_{k=1}^{\infty} y_{k}(\sigma)\left[\varphi(t) \lambda^{-t / \sigma}\right]^{k},
$$

where $\lambda>1$ is the eigenvalue of (23) linearized at the hyperbolic fixed point $(2,0), y_{1}(\sigma) \neq 0$ and $\varphi(t)$ is a $\sigma$-periodic function. Taking $\varphi(t)$ to be a positive constant, one can notice that $y_{s}(t, \sigma)$ becomes a $2 \pi \sigma i / \ln \lambda$ periodic function and that the series converges in the half plane of the complex $t$ plane $\operatorname{Re} t>R$, where $R$ is some positive constant that depends on $y_{s}$.

The fact that $y_{s}(t, \sigma)$ is analytic in $\operatorname{Re} t>R$ implies that $y_{s}(t, \sigma)$ is an entire function of the complex variable $t$. This is demonstrated as follows: Eq. (15) can be written as

$$
\begin{aligned}
y(t-\sigma, \sigma)= & 2 y(t, \sigma)-y(t+\sigma, \sigma) \\
& +\sigma^{2}\left[y^{2}(t, \sigma)-2 y(t, \sigma)\right] .
\end{aligned}
$$

This implies that $y_{s}(t, \sigma)$ is also analytic in the strip $R-\sigma$ $<\operatorname{Re} t \leqslant R$. Thus (26) allows one to "propagate" the analyticity of $y_{s}(t, \sigma)$ into the whole $t$ plane. This means that $y_{s}(t, \sigma)$ is an entire function and that the series (25) converges for all $t \in \mathbf{C}$. As a consequence, $y_{s}(t, \sigma)$ is bounded in the half plane $\operatorname{Re} t \geqslant 0$. It also implies that the power series $\chi(\tau)=2+\sum_{k=1}^{\infty} y_{k}(\sigma) \tau^{k}$ defines an entire function of $\tau$.

Suppose now that $W_{s}=W_{u}$. Then the entire function $y(t)=y_{s}(t, \sigma)$ is simultaneously a stable and an unstable solution. Time-reversibility arguments show that

$$
y(t)=\chi\left[\varphi(t) \lambda^{t / \sigma}\right]
$$

for some $\sigma$-periodic function $\varphi(t)$. One can apply here arguments of analyticity to show that $\varphi(t)$ is analytic in the complex $t$ plane. Then $y(t)$ is bounded in the semistrip Re $t \leqslant 0,0 \leqslant \operatorname{Im} t \leqslant 2 \pi \sigma / \ln \lambda$ and thus in the whole left half plane because of periodicity of $y(t)$. So, according to the Louiville theorem, $y$ is a constant and we obtain a contradiction with the assumption $W_{s}=W_{u}$.

\section{E. Onset of chaotic motion}

The fact the separatrix of (11) splits into the stable $W_{s}$ and unstable $W_{u}$ manifolds once $\sigma$ becomes positive and that these manifolds intersect giving rise to homoclinic points implies that the dynamics of the perturbed system becomes chaotic (see, for example, Ref. 32). The following arguments can be used to show that the manifolds intersect, that is, that $W_{s} \cap W_{u} \neq \emptyset$.

By continuity arguments $W_{u}$ intersects with the $\eta$ axis of the mapping $T$. Let $P$ be the point of intersection on the $(\eta, \xi)$ plane. We can always chose the unstable solution $y_{u}$ so that $P$ has coordinates $\left(y_{u}(0), \Delta_{\sigma} y_{u}(0)\right)$. Then $\Delta_{\sigma} y_{u}(0)$ $=0$. Equation (15) is time-reversible, and therefore $y_{s}(t)$ $=y_{u}(-t-\sigma)$ also satisfies (15). It is clear that

$$
\lim _{t \rightarrow+\infty} y_{u}(-t-\sigma)=\lim _{t \rightarrow-\infty} y_{u}(t-\sigma)=2,
$$

and thus $y_{u}(-t-\sigma)$ is a stable solution. Therefore the curve $\left(y_{s}(t), \Delta_{\sigma} y_{s}(t)\right)$ defines $W_{s}$. Next we show that $P \in W_{s}$. Indeed,

$$
\begin{aligned}
\left.\Delta_{\sigma} y_{s}(t)\right|_{t=0} & =\left.\frac{y_{s}(t)-y_{s}(t-\sigma)}{\sigma}\right|_{t=0} \\
& =\left.\frac{y_{u}(-t-\sigma)-y_{u}(-t)}{\sigma}\right|_{t=0} \\
& =-\left.\Delta_{\sigma} y_{u}(t)\right|_{t=0} .
\end{aligned}
$$

Therefore $\left.\Delta_{\sigma} y_{s}\right|_{t=0}=-\left.\Delta_{\sigma} y_{u}\right|_{t=0}=0$. On the other hand, $y_{s}(0)=y_{u}(-\sigma)$. This taken together with $\Delta_{\sigma} y_{u}(0)=0$ yields $y_{s}(0)=y_{u}(0)$. This implies that $P \in W_{s}$, and thus $P$ $\in W_{s} \cap W_{u}$. This intersection is transversal, as follows from the discussion below, and the angle of intersection is exponentially small in $\sigma$, thereby demonstrating that the point $P$ is a homoclinic point.

The fact that $W_{s} \cap W_{u} \neq \varnothing$ implies that $\left|y_{s}(t)-y_{u}(t)\right|$ is exponentially small in $\sigma$ for any compact interval of the real $t$ axis (see Ref. 35, Th. 4.4 for details).

\section{F. Perturbation series expansion}

We begin by constructing a formal solution of (15) as the usual perturbation series

$$
\hat{y}_{0}(t, \sigma)=\sum_{\ell=0}^{\infty} y_{0}(t) \sigma^{2 \ell},
$$

which will be called the outer expansion of (15). Substitution of (28) into (15) yields the recurrent system of ordinary differential equations

$$
\begin{aligned}
& y_{00}^{\prime \prime}(t)=y_{00}^{2}(t)-2 y_{00}(t), \\
& y_{0 \ell}^{\prime \prime}(t)=2\left(y_{00}(t)-1\right) y_{0}(t)+F_{\ell}, \quad \ell \geqslant 1,
\end{aligned}
$$

where $F_{\ell}$ is a polynomial of the functions $y_{00}(t)$, $y_{01}(t), \ldots, y_{0 \ell-1}(t)$ and their derivatives. These functions are determined in previous steps. For example, $F_{1}\left[y_{00}\right]$ $=-\frac{1}{12} y_{00}^{\prime \prime \prime}$. The first equation in (29) is Eq. (11). As we are interested in perturbation of the separatrix solution (14) of (11), we set

$$
y_{00}(t)=-1+3 \tanh ^{2} \frac{t}{\sqrt{2}} .
$$

Equations (29) with $l \geqslant 1$ are linear nonhomogeneous equations. The odd and even linearly independent solutions of the corresponding homogeneous equation are

$$
v_{1}(t)=\frac{d}{d t} y_{00}(t), \quad v_{2}(t)=v_{1}(t) \int_{0}^{t} \frac{d \xi}{v_{1}^{2}(\xi)} .
$$

It then follows from (30) that 


$$
v_{1}(t)=3 \sqrt{2} \tanh \tau \operatorname{sech}^{2} \tau,
$$

and

$$
\begin{aligned}
v_{2}(t)= & \frac{1}{24}\left[(15 \tau \tanh \tau-8) \operatorname{sech}^{2} \tau+7 \tanh ^{2} \tau\right. \\
& \left.+2 \tanh ^{2} \tau \cosh ^{2} \tau\right]
\end{aligned}
$$

where $\tau=t / \sqrt{2}$. Note that the Wronskian of $v_{1}$ and $v_{2}$ is equal to one.

In order to obtain the outer expansion (28) of an unstable solution $y_{u}(t, \sigma)$, we need, in accordance with (24), to require

$$
\lim _{t \rightarrow-\infty} y_{u}(t, \sigma)=2
$$

This boundary condition implies the following boundary conditions for $y_{0} \ell(t)$ :

$$
\lim _{t \rightarrow-\infty} y_{0}(t)=0
$$

for $\ell \geqslant 1$. Additionally, we want to shift the origin of time $t$ in such a way that

$$
y_{0}^{\prime}(0)=0 \text {. }
$$

Conditions (34) and (35) uniquely define solutions $y_{01}$, $y_{02}, \ldots$ of (29). Consider $\ell=1$. It follows from (29)-(32) that the absolute value of the function $F_{1}=-\frac{1}{12} y_{00}^{\prime \prime \prime}$ behaves as $e^{-2|\tau|}$ in the limit $t \rightarrow \infty$, because

$$
\begin{aligned}
y_{00}^{\prime \prime \prime} & =\left(y_{00}^{2}-2 y_{00}\right)^{\prime \prime} \\
& =2\left[\left(y_{00}-1\right) y_{00}^{\prime}\right]^{\prime} \\
& =2\left(y_{00}^{\prime}\right)^{2}+2 y_{00}\left(y_{00}-1\right)\left(y_{00}-2\right) .
\end{aligned}
$$

Note that $v_{1}$ and $v_{2}$ are odd and even functions, respectively, and that $\left|v_{1} F_{1}\right| \sim e^{-4|\tau|}$ as $|\tau| \rightarrow \infty$. Then there exists a unique solution of (29) (with $\ell=1$ ) given by

$$
y_{01}(t)=-v_{1}(t) \int_{0}^{t} v_{2}(\xi) F_{1} d \xi+v_{2}(t) \int_{-\infty}^{t} v_{1}(\xi) F_{1} d \xi,
$$

that satisfies (34) and (35). Indeed, all other solutions of (29) (with $\ell=1$ ) differ from (37) by the additional term $c_{1} v_{1}$ $+c_{2} v_{2}$. If either constant $c_{1}$ or $c_{2}$ differs from 0 , then one of the conditions (34) and (35) for $y_{01}(t)$ is violated. These arguments can be extended to the cases of $\ell=2,3, \ldots$ in order to demonstrate the existence and uniqueness of solutions $y_{01}, y_{02}, \ldots$ of (29) satisfying (34) and (35). Note that these solutions are even.

For $\ell=1$ we have

$$
\begin{aligned}
y_{01}(t)= & -\frac{y_{00}^{\prime}(t)}{12}\left[-\int_{0}^{t} y_{00}^{\prime}(s) y_{00}^{\prime \prime \prime \prime}(s) \int_{0}^{s} \frac{d \xi}{y_{00}^{\prime}(\xi)^{2}} d s\right. \\
& \left.+\int_{0}^{t} \frac{d s}{y_{00}^{\prime}(s)^{2}} \cdot \int_{-\infty}^{t} y_{00}^{\prime}(s) y_{00}^{\prime \prime \prime \prime}(s) d s\right],
\end{aligned}
$$

which after some algebra gives

$$
y_{01}(t)=\frac{1}{4}(7-2 \tau \tanh \tau) \operatorname{sech}^{2} \tau-\frac{15}{8} \operatorname{sech}^{4} 3 \tau .
$$

\section{G. Rescaling of variables}

The coefficients in the expansion (28) are even functions of $t$, so if (28) is convergent for some small $\sigma$ on the negative semiaxis $t \leqslant 0$, then it would define a separatrix solution to (15). However, as we observed in Sec. II D, this is not the case. This implies that the difference between $W_{s}$ and $W_{u}$ should be of the order less than $O\left(\sigma^{n}\right)$ for any $n \in \mathbf{N}$, otherwise we should be able to detect this difference in the formal expansion (28). Thus the phenomenon we are interested in is "beyond all orders" of $\sigma$.

In order to implement the approach of Ref. 3 we observe that the separatrix solution (30) to (11) has second-order poles at the points $t=i \pi / \sqrt{2}+\sqrt{2} \pi k, k \in \mathbf{Z}$. These points are also singular points for the coefficients $y_{0 l}(\sigma)$ of (28). The idea of the "asymptotic beyond all orders" approach is to scale the independent variable around a singular point, say $t=i \pi / \sqrt{2}$, and, at the same time, to scale the dependent variable so that the resulting equation is not a singularly perturbed equation in the sense of Sec. II C. This is accomplished by the transformation

$$
\begin{aligned}
& t=\frac{i \pi}{\sqrt{2}}+\sigma z, \\
& \Phi(z, \sigma)=\sigma^{2} y(t, \sigma) .
\end{aligned}
$$

Then the perturbed equation (15) (outer equation) becomes

$$
\Delta^{2} \Phi(z, \sigma)=\Phi^{2}(z, \sigma)-2 \sigma^{2} \Phi(z, \sigma),
$$

where $\Delta^{2}$ is the second-order difference operator:

$$
\Delta^{2} \Phi(z) \equiv \Phi(z+1)-2 \Phi(z)+\Phi(z-1) .
$$

Equation (40) is called the inner equation.

\section{H. Correspondence between the solutions of the outer and inner equations}

The advantage of working with the inner equation (40), instead of the original outer equation (15), is that in contrast to (15), the difference between the stable and unstable solutions is detectable in the leading-order equation

$$
\Delta^{2} \Phi(z)=\Phi^{2}(z)
$$

of (40), which is called the truncated inner equation. Next we must identify which of the solutions of (41) correspond to the stable and unstable solutions of (15). The following arguments show this correspondence on the formal level.

Consider the expansion (28) in a neighborhood of a singular point $t=i \pi / \sqrt{2}$. The leading term $y_{00}(t)$ of the outer expansion (28) has a second-order pole at this point. Then $F_{1} \equiv-\frac{1}{12} y_{00}^{\prime \prime \prime}$ has a sixth-order pole at the same point. Examining Eq. (29) for $\ell=1$ one sees that $y_{01}(t)$ has a fourthorder pole at $t=i \pi / \sqrt{2}$. Using similar arguments it can be shown that

$$
y_{0 \ell}(t) \sim \frac{a_{\ell}}{(t-i \pi / \sqrt{2})^{2(\ell+1)}}\left(\left(1+O\left(t-\frac{i \pi}{\sqrt{2}}\right)\right),\right.
$$

$$
t \rightarrow \frac{i \pi}{\sqrt{2}}
$$


where $a_{\ell}$ for $\ell=0,1,2, \ldots$ are certain real numbers.

Taking into account (39) one obtains

$$
\begin{aligned}
\hat{\Phi}(z, \sigma) & =\sigma^{2} \hat{y}_{0}(t, \sigma) \\
& =\sum_{\ell=0}^{\infty} y_{0 \ell}(t) \sigma^{2 \ell+2} \\
& =\sum_{\ell=0}^{\infty} \frac{a \ell \sigma^{2 \ell+2}}{\sigma^{2 \ell+2} z^{2 \ell+2}}+(\text { terms of order } \sigma) \\
& =\hat{\Phi}_{0}(z)+(\text { terms of order } \sigma),
\end{aligned}
$$

where $\hat{\Phi}(z, \sigma)$ is a formal solution to (40), and the formal power series

$$
\hat{\Phi}_{0}(z)=\sum_{\ell=0}^{\infty} \frac{a_{\ell}}{z^{2 \ell+2}}
$$

is a formal solution of (41).

Therefore, the actual solutions of (41) that have the asymptotic expansion (44) in the right (left) half plane of the complex $z$ plane correspond to the stable (unstable) solutions to (15). These solutions $\Phi_{s}(z)$ and $\Phi_{u}(z)$ are called, respectively, stable and unstable solutions to (41). As we will see below, these solutions are uniquely defined and do not coincide with each other.

Rigorous arguments to demonstrate the correspondence between stable and unstable solutions of (15) and of (41) can be based on the fact that (28) is the asymptotic expansion of $y_{s}(t, \sigma)$ and $y_{u}(t, \sigma)$ as $\sigma \rightarrow 0$ in the right and in the left half planes of the complex $t$ plane, respectively. A paper addressing some theoretical aspects of the inner-outer correspondence is in progress now.

\section{STOKES PHENOMENON, EXPONENTIAL ASYMPTOTICS, AND APPROXIMATION}

\section{A. Irregular singular points and the Stokes phenomenon}

In order to find the difference between the solutions $y_{s}(t, \sigma)$ and $y_{u}(t, \sigma)$ of the outer equation (15), we need to determine the difference between the corresponding solutions of the inner equation (41). To accomplish this we will study the Stokes phenomenon for the inner equation. To this end we will start with a brief review of some concepts of the analytic theory of differential equations that we will apply to the nonlinear difference equation (41) (some facts on the analytic theory of difference equations can be found in Ref. 36).

Consider a matrix differential equation

$$
Y^{\prime}(z)=A(z) Y(z),
$$

where $A(z)$ has a pole at some point $z_{0}$. The point $z_{0}$ is called a regular singular point of (45) if every solution $Y(z)$ of (45) has no more than a power growth as $z \rightarrow z_{0}$. In other words, $Y(z)$ can have at most a pole type singularity at $z_{0}$ combined with some branching. In the opposite case $z=z_{0}$ is called an irregular singular point of (45). In this case solutions can have exponential growth or decay as $z \rightarrow z_{0}$ (there is no standard terminology yet).

Very often the singular point $z_{0}$ is placed at $\infty$. We call $z_{0}=\infty$ a singular point of $(45)$ if $A(z)=z^{N}\left(A_{0}+A_{1} z^{-1}\right.$ $+\cdots$ ), where $A_{0} \neq 0$ and $N \geqslant-1$ (see, for example, Ref. 37, Chap. 4). Suppose that a formal power series $\hat{Y}(z)$ $=\sum_{k=0}^{\infty} Y_{k} z^{-k}$ satisfies a nonhomogeneous equation (45), where the nonhomogeneous term is analytic at $\infty$. Then, the case $z_{0}=\infty$ is a regular singular point, $\hat{Y}(z)$ is convergent (has nonzero radius of convergence). Generically, in the case of an irregular singularity $\hat{Y}(z)$ defines only an asymptotic expansion (as $z \rightarrow \infty)$ of some actual solution. This expansion is valid only in a certain sector of the complex $z$ plane and fails outside this sector. This phenomenon is known as the Stokes phenomenon.

Example 1. Consider a solution of

$$
y^{\prime}(x)=-y(x)+\frac{1}{x}
$$

satisfying $y\left(r e^{i \pi}\right) \rightarrow 0$ as $r \rightarrow \infty$. This solution is given by

$$
y(x)=e^{-x} \int_{e^{i \pi_{\infty}}}^{x} \frac{e^{t}}{t} d t=e^{-x} \operatorname{Ei}(x),
$$

where $\operatorname{Ei}(x)$ is the standard integral exponential function. Integration by parts and standard estimates (see, for example, Ref. 37, Chap. 3) show that

$$
y(x) \sim \sum_{n=0}^{\infty} \frac{n !}{x^{n+1}}, \quad x \rightarrow \infty
$$

and this asymptotic expansion is valid in the sector $S:|\arg x-\pi|<\frac{3}{2} \pi$. However,

$$
y(x)-y\left(x e^{-2 \pi i}\right)=-e^{-x} \oint_{|t|=\text { const }} \frac{e^{t}}{t} d t=-2 \pi i e^{-x},
$$

so that the expansion (47) gains the exponential term $-2 \pi i e^{-x}$, which grows once $x$ crosses the ray $\arg x$ $=-\pi / 2$. Therefore (47) cannot be valid outside $S$.

The appearance of exponentially small terms, which lead to the breakdown of asymptotic expansions, is an essential feature of the Stokes phenomenon. As we will see below, these exponential terms cause splitting of separatrices. At the same time they can be used to approximate the stable and unstable solutions.

\section{B. The Stokes phenomenon for the inner equation}

Irregular singularities for the difference equations can be defined in much the same manner as for differential equations. An effective technique to study the Stokes phenomenon for nonlinear equations is the Borel-Laplace summation (also known as a particular case of resurgence, see, for example, Ref. 38). We will now apply this method to the inner equation (41).

The inverse Laplace transform $\mathscr{B}^{-1}$ transforms (41) into the nonlinear convolution equation

$$
2(\cosh p-1) V(p)=V(p) * V(p),
$$




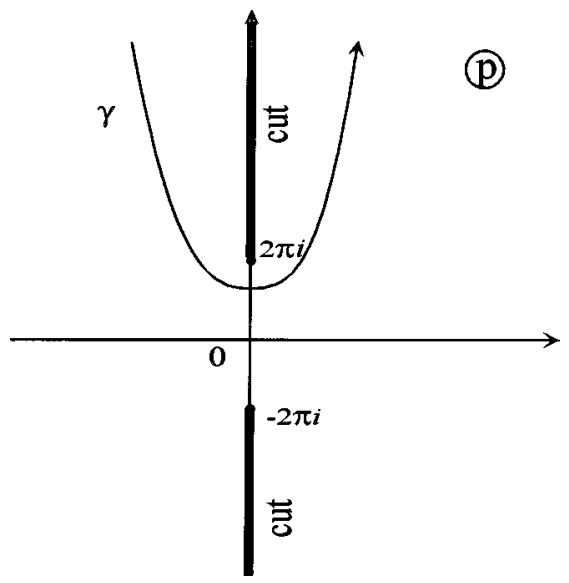

FIG. 2. Contour of integration $\gamma$ for $\Phi_{-}(z)$ in the cut $p$ plane.

where $\quad V(p)=\left[\mathscr{B}^{-1} \Phi\right](p)$ and $V(p)^{*} W(p) \stackrel{\text { def }}{=} \int_{0}^{p} V(p$ $-\tau) W(\tau) d \tau$.

Theorem 1. There exists a unique solution $V(p)$ of (48) that is an odd function and is analytic in the whole $p$ plane with two vertical cuts: from $p=2 \pi i$ upwards and from the point $p=-2 \pi i$ downwards (see Fig. 2). This solution is majorized by $C e^{|p|}$ along each nonvertical ray in the cut plane with the appropriate constant $C>0$.

Convolution equations of the type (48), which also arise from inverse Laplace transform of differential equations, have been investigated in Ref. 9. The arguments of Theorem 3 in Ref. 9 can be used to prove the Theorem 1 .

Theorem 1 implies that the Laplace transform $\Phi(z)$ $=\mathscr{B} V$ is well-defined and yields a solution to (41). It is well known that the Taylor expansion of $V(p)$ at $p=0$ defines the asymptotic expansion of $\Phi(z)$ as $z \rightarrow \infty$. This asymptotic expansion is a formal power series solution of (41) in even powers of $1 / z$. Since (41) possesses a unique nontrivial formal power series solution $\hat{\Phi}_{0}$ in negative powers of $z^{2}$,

$$
\Phi(z) \sim \hat{\Phi}_{0}(z), \quad z \rightarrow \infty, \quad \operatorname{Re} z>0 .
$$

So,

$$
V(p)=\sum_{\ell=1}^{\infty} \frac{a_{\ell}}{(2 \ell-1) !} p^{2 \ell-1},
$$

where $a_{\ell}$ are the coefficients of $\hat{\Phi}_{0}(z)$.

The function $V(p)$ is called the formal Borel transform of $\hat{\Phi}_{0}(z), V=\mathscr{B} \hat{\Phi}_{0}$, and the solution $\Phi(z)=\mathscr{C} \mathscr{B} \hat{\Phi}_{0}(z)$ is known as the Borel-Laplace sum of $\hat{\Phi}_{0}$. It is the only solution of (41) satisfying (49). Using the Borel-Laplace summation, we construct two different solutions

$$
\Phi_{s}(z)=\int_{0}^{\infty} e^{-p z} V(p) d p
$$

and

$$
\Phi_{u}(z)=\int_{0}^{-\infty} e^{-p z} V(p) d p .
$$

These solutions possess the asymptotic expansion $\hat{\Phi}_{0}(z)$ in the right and left half planes, respectively. In fact, these
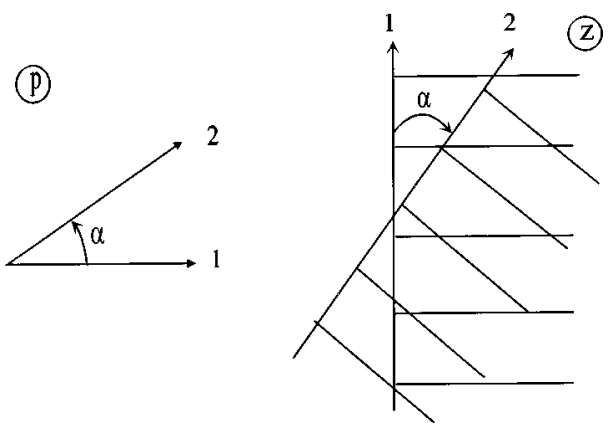

FIG. 3. The simple relation between rotation of the ray of integration in the $p$ plane and rotation of the corresponding half plane of analyticity of the Laplace transform in the $z$ plane.

asymptotic expansions are valid in wider sectors $|\arg z|<\pi$ $-\delta$ and $\delta<\arg z<2 \pi-\delta$, respectively, where $\delta$ is an arbitrary small positive number. This can be verified by rotating the ray of integration, say, for $\Phi_{s}(z)$ in (51) from $\arg p$ $=-\pi / 2+\delta$ to $\arg p=\pi / 2-\delta$. Figure 3 illustrates the simple relation between rotation of the ray of integration in the $p$ plane and rotation of the corresponding half plane of analyticity of the Laplace transform in the $z$ plane.

Although solutions $\Phi_{s}(z)$ and $\Phi_{u}(z)$ have the same asymptotics $\hat{\Phi}_{0}(z)$ in two disjoint sectors $|\arg z \pm \pi / 2|<\pi / 2$ $-\delta$, they do not coincide with each other. Indeed, $\Phi_{s}(z)$ $\equiv \Phi_{u}(z)$ would mean that $\Phi_{s}(z)$ is an analytic function at $z=\infty$ (see, for example, Ref. 37, Chap. 3). Hence the formal series (44) would be convergent. This then would imply that $V(p)$ is an entire function. The following arguments from Ref. 9 show that this cannot be the case.

Indeed, one can show that the series (44) is signalternating. This implies that the function $-i V(p)$ is positive on $[0, i a)$, where $i a$ is the singularity closest to the origin on the positive imaginary semiaxis. Theorem 1 implies that $a$ $\geqslant 2 \pi$. Suppose that $p=2 \pi i$ is not a singular point of $V(p)$. Then the convolution $\left.V(p)^{*} V(p)\right|_{p=2 \pi i}<0$, while the lefthand side of (48) is zero at $p=2 \pi i$. This yields a contradiction which shows that $p=2 \pi i$ is a singular point of $V(p)$. In fact, the points $p= \pm 2 \pi i k, k \in \mathbf{Z} \backslash\{0\}$ on the sheets of the corresponding Riemann surface are the singular points of $V(p)$.

Thus the rays of integration in (51) cannot be rotated beyond $\arg p= \pm \pi / 2$, implying that $\Phi_{s}(z)$ and $\Phi_{u}(z)$ do not coincide. Moreover, the asymptotics $\hat{\Phi}_{0}(z)$ is not valid for $\Phi_{s}(z)$ and $\Phi_{u}(z)$ beyond the sectors mentioned above. This is the Stokes phenomena for Eq. (41) at $z=0$.

Let $\Phi_{ \pm}(z)=\Phi_{s}(z)-\Phi_{u}(z)$ when $\pm \operatorname{Im} z>0$. The subscript indicates the sign of the imaginary part of $z$. Then the difference between the stable and unstable solutions of (41) is given by

$$
\Phi_{-}(z)=\int_{\gamma} e^{-p z} V(p) d p,
$$

where the contour $\gamma$ encircles the positive imaginary semiaxis from the left (see Fig. 2). This integral (52) is well defined only if $\operatorname{Im} z<0$. The leading term of the asymptotics 
of (52) as $\operatorname{Im} z \rightarrow \infty$ is determined by the singularity of $V(p)$ at the point $p=2 \pi i$. This is the term that we will focus on in the following subsections.

\section{Exponential expansion for the inner equation}

In order to obtain more information about $\Phi_{-}(z)$ we look for a solution of (41) with the form

$$
\stackrel{\circ}{\Phi}(z)=\hat{\Phi}_{0}(z)+\hat{\Phi}_{1}(z) e^{-2 \pi i z}+\hat{\Phi}_{2}(z) e^{-4 \pi i z}+\cdots,
$$

where $\hat{\Phi}_{j}(z), j=0,1,2, \ldots$, are formal power series in $z^{-1}$. Substituting into (41) we obtain

$$
\begin{aligned}
& \Delta^{2} \hat{\Phi}_{0}(z)=\hat{\Phi}_{0}^{2}(z) \\
& \Delta^{2} \hat{\Phi}_{1}(z)=2 \hat{\Phi}_{0}(z) \hat{\Phi}_{1}(z), \\
& \Delta^{2} \hat{\Phi}_{k}(z)=2 \hat{\Phi}_{0}(z) \hat{\Phi}_{k}(z)+\hat{\Phi}_{k-1}^{2}(z), \quad k \geqslant 2 .
\end{aligned}
$$

Our choice of the expansion (53) is motivated by the following fact: if an ODE with the irregular singular point $z=\infty$ has a formal power solution $\hat{\Phi}_{0}(z)$ and if $p=2 \pi i$ is a singular point of $\mathscr{B} \hat{\Phi}_{0}$, then it has a formal solution of the form (53). Suppose that $p=2 \pi i$ is the first singularity of $\mathscr{B} \hat{\Phi}_{0}$ in the positive imaginary direction. Then there exists analytic functions $\Phi_{k}(z)$, such that:

(1) $\Phi_{k}(z) \sim \hat{\Phi}_{k}(z)$ as $z \rightarrow \infty, z \in S$ where $S$ is some sector in the lower half-plane which contains the negative imaginary semiaxis.

(2) For every $c \in \mathbf{C}$, the series $\Phi(z)=\Phi_{0}(z)$ $+c \Phi_{1}(z) e^{-2 \pi i z}+c^{2} \Phi_{2}(z) e^{-4 \pi i z}+\cdots$ is convergent for sufficiently large $|z|, z \in S$.

(3) The functions $\Phi(z)$ form a one-parameter family of solutions of the given equation. ${ }^{39}$

Every solution from this family has the same power series asymptotic expansion $\hat{\Phi}_{0}(z)$ in $S$. The difference between two such solutions is exponentially small in $S$ as $z$ $\rightarrow \infty$ and is given by constant $\times \Phi_{1} e^{-2 \pi i z}$. In what follows, we apply the same arguments to the solutions $\Phi_{s}(z)$ and $\Phi_{u}(z)$ of the difference equation (41).

The first equation in (54), which is Eq. (41), is nonlinear. It possesses a unique formal power series solution in $z^{-2}$ :

$$
\hat{\Phi}_{0}(z)=\frac{6}{z^{2}}-\frac{5}{4 z^{4}}+\cdots .
$$

The remaining equations are linear and have equal homogeneous parts.

In order to find the leading term of a solution to the second equation (54), we replace $\hat{\Phi}_{0}(z)$ by its leading term $\left(6 / z^{2}\right)$. This yields

$$
\Delta^{2} \hat{\Phi}_{1}(z)=\frac{12}{z^{2}} \hat{\Phi}_{1}(z)
$$

which possesses two formal power series (in $z^{-1}$ ) solutions with leading terms $z^{4}$ and $z^{-3}$, respectively. Therefore, the second equation (54) also possesses two solutions $\hat{\Phi}_{1}^{\text {even }}(z)$, $\hat{\Phi}_{1}^{\text {odd }}(z)$ with the corresponding leading terms. For large val- ues of $|z|$ the even solution $\hat{\Phi}_{1}^{\text {even }}(z)$ dominates the odd solution $\hat{\Phi}_{1}^{\text {odd }}(z)$. Thus we will only consider $\hat{\Phi}_{1}^{\text {even }}(z)$ and will denote it by $\hat{\Phi}_{1}(z)$. In fact, one can find formal solutions $\hat{\Phi}_{2}(z), \hat{\Phi}_{3}(z), \ldots$ to all equations (54). However, in this paper we restrict our attention to the first two terms in the exponential expansion (53).

The solutions $\Phi_{s}(z)$ and $\Phi_{u}(z)$ of (41) have the same power series asymptotic expansion along the negative imaginary semiaxis, but differ in exponential small terms. The leading asymptotic term of their difference $\Phi_{-}(z)$ is proportional to $\hat{\Phi}_{1}(z) e^{-2 \pi i z}$. (The higher-order exponential terms are exponentially small with respect to $\Phi_{1}(z) e^{-2 \pi i z}$ in the limit $\operatorname{Im} z \rightarrow \infty$.) This leading term is $c z^{4} e^{-2 \pi i z}$ where $c$ is a constant prefactor.

\section{Evaluation of $c$}

According to (48), $V(p)$ is an odd function and is realvalued along the real axis as all coefficients $a_{l}$ are real. Therefore we obtain

$$
\Phi_{u}(-z)=\Phi_{s}(z), \quad \text { and } \Phi_{s}(\bar{z})=\bar{\Phi}_{s}(z) .
$$

If $\zeta \in \mathbf{R}$, then

$$
\Phi_{u}(-i \zeta)=\Phi_{s}(i \zeta)=\bar{\Phi}_{s}(-i \zeta)
$$

so that

$$
\Phi_{ \pm}(z)=\Phi_{s}(z)-\Phi_{u}(z)=-2 i \operatorname{Im} \Phi_{u}(z)
$$

is purely imaginary when $\operatorname{Re} z=0$. Therefore, the constant prefactor $c$ is purely imaginary. Thus

$$
i \operatorname{Im} \Phi_{u}(z) \sim-\frac{c}{2} z^{4} e^{-2 \pi i z}, \quad z \rightarrow \infty, \quad \arg z=-\frac{\pi}{2} .
$$

Consider the solution $\Phi_{u}(z)$ to Eq. (41) near the singular point $z_{0}=\infty$. It possesses the asymptotics $\hat{\Phi}_{0}(z)$ in the sector $-2 \pi+\delta<\arg z<-\delta$, where $\delta$ is an arbitrary small positive number. However, as $\arg z$ becomes greater than zero, the path of integration in the integral representation (51) of $\Phi_{u}(z)$ will cross the positive imaginary semiaxis in the $p$ plane. Then the singularities $2 \pi i k, k=1,2, \ldots$, of $V(p)$ will contribute terms that are proportional to $e^{-2 \pi i k z}$. These terms destroy the power series asymptotic expansion $\hat{\Phi}_{0}(z)$ of $\Phi_{u}(z)$ if $\arg z>0$.

The terms, mentioned above, are present in the asymptotics of $\Phi_{u}(z)$ when $\arg z<0$. However, they are exponentially small in the lower half plane and thus do not affect power series asymptotics $\hat{\Phi}_{0}(z)$ there. Let us consider the leading exponential term of $\Phi_{u}(z)$ that has the form $b z^{4} e^{-2 \pi i z}$. Here the prefactor $b$ varies according to the direction in which $z$ approaches infinity [otherwise, the asymptotics $\Phi_{u}(z) \sim \hat{\Phi}_{0}(z), z \rightarrow \infty$ would not be valid for $\arg z$ $<-\pi]$. One can also show that in the limit $z \rightarrow \infty$ 


$$
b=\left\{\begin{array}{l}
0, \quad-\pi<\arg z<-\frac{\pi}{2} \\
-\frac{c}{2}, \quad \arg z=-\frac{\pi}{2} \\
-c, \quad-\frac{\pi}{2}<\arg z<0 .
\end{array}\right.
$$

The line $\operatorname{Im} z=-\pi / \sqrt{2} \sigma$ in the $z$ plane corresponds to the real line axis in the $t$ plane. As we have seen, the prefactor $b$ varies along this line, reaching the value 0 as $\operatorname{Re} z \rightarrow-\infty$ and $-c$ as $\operatorname{Re} z \rightarrow+\infty$. Unfortunately, theory provides no general results on this issue (see, however, some discussion on the smoothing of Stokes phenomena in Refs. 40 and 41), so we are compelled to use numerical simulations in modeling the exponentially small terms of $\Phi_{u}(z)$.

The constant $c$ in (59) is determined by Eq. (41) and is known as the Stokes constant. The power prefactor $z^{4}$ to the exponential term $e^{-2 \pi i z}$ indicates the order of singularity of $V(P)=\mathscr{B} \hat{\Phi}_{0}(z)$ at the point $p=2 \pi i$. Thus the leading singular term of $V(p)$ is of order $O(p-2 \pi i)^{-5}$. It is known that if $V(p) \sim K(p-2 \pi i)^{-1}$, then the Stokes constant is equal to the residue at this pole (see Ref. 19), i.e., $c$ $=2 \pi i K$. Therefore,

$$
c=2 \pi i \lim _{\substack{p \rightarrow 2 \pi i \\ \operatorname{Im} p<2 \pi i}}(p-2 \pi i) \mathscr{B}\left[z^{-4} \hat{\Phi}_{0}(z)\right](p) .
$$

Our calculations show (see Sec. IV D) that $c=2 \pi i K$, where $K \simeq 7374$. To calculate $K$, we have used the same heuristic algorithm as used in Ref. 15 and 16, which can be rigorously justified by results of Ref. 9 .

\section{E. Exponential expansions for the outer equation}

As it has been shown above, the exponential expansion (53) for the inner equation (41) is capable of capturing the exponentially small $\Phi_{s}(z)-\Phi_{u}(z)$ on the ray $\arg z$ $=-\pi / 2$. The corresponding expansion for the outer equation (15) is

$$
\begin{aligned}
\hat{y}(t, \sigma)= & \hat{y}_{0}(t, \sigma)+\hat{y}_{1}(t, \sigma) e^{-2 \pi i t / \sigma}+\hat{y}_{2}(t, \sigma) e^{-4 \pi i t / \sigma} \\
& +\cdots
\end{aligned}
$$

One can hope therefore that (61) will also be able to capture the exponentially small $y_{s}(t, \sigma)-y_{u}(t, \sigma)$, where $t$ $\in(0, i \pi / \sqrt{2})$. We assume $\hat{y}_{j}(t, \sigma), j=0,1,2, \ldots$ in $(61)$ to be a formal power series in $\sigma$. In particular, $\hat{y}_{0}(t, \sigma)$ is the outer expansion (28). The other $\hat{y}_{j}(t, \sigma)$ satisfy the recurrent system of difference equations that can be obtained by substituting (61) into (15). The coefficients $\hat{y}_{j}(t, \sigma)$ correspond to the coefficients $\hat{\Phi}_{j}(z)$ in expansion (53). Here we would like to mention that the formal series $\hat{y}_{1}(0, \sigma)$ for the discretized pendulum (standard map) has been derived and discussed by Lazutkin in Ref. 24.

The coefficient $\hat{y}_{1}(t, \sigma)$ of the leading exponential term in (61) satisfies the equation

$$
\frac{\Delta^{2} \hat{y}_{1}(t, \sigma)}{\sigma^{2}}=2\left(\hat{y}_{0}(t, \sigma)-1\right) \hat{y}_{1}(t, \sigma) .
$$

In order to consider the leading term $y_{10}(t)$ of $\hat{y}_{1}(t, \sigma)$, we replace $\hat{y}_{0}(t, \sigma)$ in (62) by its leading term

$$
y_{00}(t)=-1+3 \tanh ^{2} \frac{t}{\sqrt{2}} .
$$

Then,

$$
y_{10}^{\prime \prime}(t)=2\left(y_{00}(t)-1\right) y_{10}(t) .
$$

Equation (64) was considered above and its odd and even linearly independent solutions $v_{1}(t)$ and $v_{2}(t)$ are given by (32) and (33), respectively.

Our aim is to use (61) to approximate $y_{u}(t, \sigma)$. In particular, we want to find the "first-order" approximation which is based on the first two terms of (61). Now the problem is how to find the correct linear combination

$$
y_{10}(t)=c_{1} v_{1}(t)+c_{2} v_{2}(t)
$$

for this approximation. This is done below by matching the leading exponential terms $i \operatorname{Im} \Phi_{u}(z)=-\frac{1}{2}\left(\Phi_{s}(z)-\Phi_{u}(z)\right)$ and $\sigma^{2} y_{10}(t) \exp (-2 \pi i t / \sigma)$ of $\Phi_{u}(z)$ and $y_{u}(t, \sigma)$, respectively.

\section{F. Matching of exponential terms}

The equation (62) for $\hat{y}_{1}(t, \sigma)$ corresponds to the second equation (54) for $\hat{\Phi}_{1}(z)$. Consider functions $v_{1}(t)$ and $v_{2}(t)$ in a neighborhood of the point $t=i \pi / \sqrt{2}$. First, we would like to match $v_{1}(t)$ and $v_{2}(t)$ with the appropriate solutions of Eq. (54). Let $\tau=t / \sqrt{2}=i \pi / \sqrt{2}$. If one introduces $\delta=\tau$ $-i \pi / 2$, then (32) implies

$$
v_{1}(t)=3 \sqrt{2} \frac{\sinh \tau}{\cosh ^{3} \tau}=-3 \sqrt{2} \frac{\cosh \delta}{\sinh ^{3} \delta} .
$$

Here, $\delta=\sigma z / \sqrt{2}$, see (39). Similarly, we obtain from (33) after some algebra

$$
\begin{aligned}
v_{2}(t)= & \frac{1}{24}\left[-15\left(\delta+\frac{i \pi}{2}\right) \frac{\cosh \delta}{\sinh ^{3} \delta}+9 \operatorname{coth}^{2} \delta+\frac{8}{\sinh ^{2} \delta}\right. \\
& \left.-2 \cosh ^{2} \delta \operatorname{coth}^{2} \delta\right] \\
= & -i \pi \frac{5}{16} \frac{\cosh \delta}{\sinh ^{3} \delta}-\frac{1}{24 \sinh ^{2} \delta}[15 \delta \operatorname{coth} \delta \\
& \left.-9 \cosh ^{2} \delta-8+2 \cosh ^{4} \delta\right] \\
= & i \pi \frac{5}{48} v_{1}(t)-\frac{1}{24 \sinh ^{2} \delta}\left[15 \delta \operatorname{coth} \delta+2 \sinh ^{4} \delta\right. \\
& \left.-5 \sinh ^{2} \delta-15\right] .
\end{aligned}
$$

Observe that the odd solution $v_{1}(t)$ of (64) remains odd with respect to the point $t=i \pi / \sqrt{2}$, while the even solution $v_{2}(t)$ splits into the linear combination (67) of the even and odd solutions. 
The leading term of $v_{1}(t)$ at $t=i \pi / \sqrt{2}$ is of the order $O\left(\delta^{-3}\right)=O\left(2^{3 / 2} / \sigma^{3} z^{3}\right)$. It corresponds to the solution $\hat{\Phi}_{1}^{\text {odd }}(z)$ of the second equation of (54). But this solution is subdominant to the other solution $\hat{\Phi}_{1}^{\text {even }}(z)$. We need to find what solution $y_{10}(t)$ of (64) corresponds to the solution $\hat{\Phi}_{1}^{\text {even }}(z)$. First, it must be an even function with respect to $t=i \pi / \sqrt{2}$, and, second, its leading term should be of order $O\left(\delta^{4}\right)$ as $t \rightarrow i \pi / \sqrt{2}$.

Let us take

$$
y_{10}(t)=c_{2}\left[v_{2}(t)-i \pi \frac{5}{48 \sqrt{2}} v_{1}(t)\right],
$$

where the constant $c_{2}$ is to be determined later. We want to check the second condition. The expression in the square brackets in (67) has the Taylor expansion

$$
\begin{gathered}
15 \delta \text { coth } \delta-5 \sinh ^{2} \delta+2 \sinh ^{4} \delta-15 \\
=0+0 \cdot \delta^{2}+0 \cdot \delta^{4}+\frac{8}{7} \delta^{6}+\cdots .
\end{gathered}
$$

It is not a coincidence that the expansion (69) starts with the term $O\left(\delta^{6}\right)$. As we will see below, this is a necessary condition to match $\hat{y}_{1}(t)$ with $\hat{\Phi}_{1}(z)$, i.e., to match the inner and outer exponential terms.

Equations (66)-(69) imply

$$
\begin{aligned}
y_{10}(t) & =-\frac{c_{2}}{24} \cdot \frac{8}{7} \delta^{4}\left(1+O\left(\delta^{2}\right)\right) \\
& =-\frac{c_{2}}{21} \cdot \frac{\sigma^{4}}{4} \cdot z^{4}\left(1+O\left(\delta^{2}\right)\right) .
\end{aligned}
$$

Now use (39) to match exponential terms in $y_{u}(t, \sigma)$ and $\Phi_{u}(z)$ for $t$ approaching $i \pi / \sqrt{2}$ from below:

$$
\sigma^{2} y_{10}(t) \exp \left(-\frac{2 \pi i t}{\sigma}\right) \sim i \operatorname{Im} \Phi_{u}(z)
$$

Here $t=i \pi / \sqrt{2}+\sigma z$ and hence $\arg z=-\pi / 2$. According to (58),

$$
-\frac{c_{2}}{84} \sigma^{6} z^{4} \exp \frac{\sqrt{2} \pi^{2}}{\sigma}=-\frac{c}{2} z^{4} .
$$

Thus

$$
c_{2}=\frac{42 c}{\sigma^{6}} \exp \frac{-\sqrt{2} \pi^{2}}{\sigma}=\frac{84 \pi i K}{\sigma^{6}} \exp \frac{-\sqrt{2} \pi^{2}}{\sigma} .
$$

If we denote

$$
c_{1}=i \pi \frac{5}{48 \sqrt{2}} c_{2}
$$

we obtain the unique solution (65) of Eq. (64) that matches $\operatorname{Im} \Phi_{u}(z)$ :

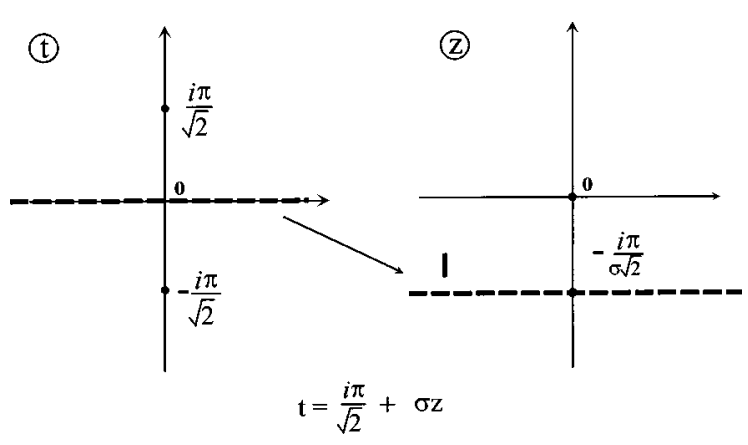

FIG. 4. The real $t$ axis corresponds to the line $\ell$ in the complex $z$ plane.

$$
\begin{aligned}
y_{10}(t)= & c_{1} v_{1}(t)+c_{2} v_{2}(t) \\
= & \frac{c_{2}}{24}\left[(15(\tau-i \pi / \sqrt{2}) \tanh \tau-8) \operatorname{sech}^{2} \tau\right. \\
& \left.+7 \tanh ^{2} \tau+2 \tanh ^{2} \tau \cosh ^{2} \tau\right] .
\end{aligned}
$$

\section{G. Approximation of the unstable solution}

The unstable solution $y_{u}(t, \sigma)$ admits a good approximation by the formal series (28) for negative values of $t$ and small positive $\sigma$. However, this approximation fails for sufficiently large positive values of $t$. Therefore higher-order terms from the series (61) must be included. The following arguments discuss the correct choice of these terms.

Let us consider the problem of approximation of $y_{u}(t, \sigma)$ as the problem of constructing analytic continuation of $y_{u}(t, \sigma)$ from the left part of the strip $T:|\operatorname{Im} t|<\pi / \sqrt{2}$ into its right part. Since $y_{u}(t, \sigma)$ is real valued for $t \in \mathbf{R}$ hence $y_{u}(\bar{t}, \sigma)=\bar{y}_{u}(t, \sigma)$. If $\gamma_{ \pm} \in T$ are complex-conjugated contours, $t>0$ and $y_{u}^{+}(t, \sigma), y_{u}^{-}(t, \sigma)$ are the corresponding analytic continuations of $y_{u}(t, \sigma)$ from the left part of $T$ to $t$, then

$$
y_{u}(t, \sigma)=\frac{1}{2}\left(y_{u}^{+}(t, \sigma)+y_{u}^{-}(t, \sigma)\right)=\operatorname{Re} y_{u}^{+}(t, \sigma) .
$$

The right-hand side of this equation can be obtained by requiring that the contour $\gamma_{+}$pass close enough to $t$ $=i \pi / \sqrt{2}$ that one can match the exponentially small terms of $y_{u}^{+}(t, \sigma)$ and of $\Phi_{u}(z)$. Then, according to (74),

$$
\dot{y}(t, \sigma)=\hat{y}_{0}(t, \sigma)+\operatorname{Re}\left[\sum_{k=0}^{\infty} \hat{y}_{k}(t, \sigma) e^{-2 \pi i k t / \sigma}\right] .
$$

This is the formal exponential expansion of solutions of the outer equation (15) for real values of $t$.

Note that $c_{2}$ is a purely imaginary constant and $c_{1} \in \mathbf{R}$. Then the leading part of the first exponential term in (75) is

$$
\operatorname{Re}\left[y_{10}(t) e^{2 \pi i t / \sigma}\right]=c_{1} v_{1}(t) \cos \frac{2 \pi t}{\sigma}-i c_{2} v_{2}(t) \sin \frac{2 \pi t}{\sigma} .
$$

The factors $c_{1}$ and $c_{2}$, defined in (71) and (72) by matching the inner and outer exponential terms, are valid for $t$ $\in[0, i \pi / \sqrt{2})$. However, when $t$ runs along the real axis, the corresponding value of $z=(t / \sigma)-(i \pi / \sigma \sqrt{2})$ runs along the line $\ell: \operatorname{Im} z=-\pi / \sigma \sqrt{2}$ (see Fig. 4). Therefore, the factors $c_{1}$ and $c_{2}$ for $t \in \mathbf{R}$ are determined by the exponentially small term of $\Phi_{u}(z)$ on the line $\ell$. As it was discussed above, this 
term has the form $b(\arg z) z^{4} e^{-2 \pi i z}$, where $b(-\pi / 2)$ $=-c / 2$ and $b(\alpha)$ varies from 0 as $\alpha \rightarrow-\pi$ to $-c$ as $\alpha$ $\rightarrow 0, \quad z \in \ell$. tion

The above arguments suggest the following representa-

$$
\operatorname{Re}\left[y_{10}(t) e^{2 \pi i t / \sigma}\right]=M(t, \sigma) B(t, \sigma),
$$

where

$$
\begin{aligned}
& B(t, \sigma)=v_{2}(t) \sin 2 \pi t / \sigma-\frac{5}{48 \sqrt{2}} v_{1}(t) \cos \frac{2 \pi t}{\sigma}, \\
& M(t, \sigma)=S(t, \sigma) \frac{84 \pi K}{\sigma^{6}} e^{-\frac{\sqrt{2} \pi^{2}}{\sigma}} .
\end{aligned}
$$

Here $S(t, \sigma)$ is determined by $b(\arg z)$. We call it the switching function, since $S(t, \sigma) \rightarrow 0$ as $t \rightarrow-\infty, S(t, \sigma) \rightarrow 2$ as $t \rightarrow+\infty$, and $S(0, \sigma)=1$.

The exponential expansion (75) is used to construct what we call the analytical approximation to the unstable solution $y_{u}(t, \sigma)$. The first-order approximation $\tilde{y}(t, \sigma)$ to the unstable solution $y_{u}(t, \sigma)$ that we discuss in the next section is chosen as

$$
\tilde{y}(t, \sigma)=y_{00}(t)+\sigma^{2} y_{01}(t)+M(t, \sigma) B(t, \sigma) .
$$

\section{COMPARISON WITH NUMERICAL RESULTS}

The first-order analytical approximation of the unstable solution $y_{u}(t, \sigma)$ to the perturbed equation (15) has been developed in the previous sections. From this approximation it is straightforward to construct an analytical approximation for the stable and unstable manifolds of the Hénon map. The discussion in this section focuses on the functional form of this approximation and compares the results obtained from this approximation with the numerically determined manifolds.

\section{A. The stable and unstable manifolds of the Hénon map}

As discussed in Sec. II B the Hénon map possesses two period-one periodic orbits (fixed points) that result from a saddle-center bifurcation at a parameter value of $\sigma=0$ ( $\lambda$ $=-1)$. The first of these periodic orbits is stable in the parameter range $0<\sigma<\sqrt{2}(-1<\lambda<3)$ and the second is unstable for all positive values of the parameter $\sigma>0(\lambda>$ $-1)$. This unstable periodic orbit possesses both a stable and unstable manifold. All orbits on the stable (unstable) manifold are asymptotic in the future (past) to the unstable periodic orbit.

Analytical approximations for these two manifolds are readily constructed from the first-order analytical approximation of the unstable solution $y_{u}(t, \sigma)$ to the perturbed equation (15) obtained in the previous section. The approximation for the unstable manifold is given by

$$
\begin{aligned}
& \left(\tilde{u}_{u}(t, \sigma), \tilde{v}_{u}(t, \sigma)\right) \\
& \quad=\left(\left(\frac{\sigma^{2}}{1-\sigma^{4}}\right) \tilde{y}_{u}(t, \sigma),\left(\frac{\sigma^{2}}{1-\sigma^{4}}\right) \tilde{y}_{u}(t-\sigma, \sigma)\right)
\end{aligned}
$$

and the approximation for the stable manifold is obtained using time-reversal symmetry (see Sec. II E)

$$
\left(\tilde{u}_{s}(t, \sigma), \tilde{v}_{s}(t, \sigma)\right)=\left(\tilde{v}_{u}(-t, \sigma), \tilde{u}_{u}(-t, \sigma)\right) .
$$

The functional form of the approximation for the unstable manifold is

$$
\begin{aligned}
\tilde{u}_{u}(t, \sigma) & =\tilde{v}_{u}(t+\sigma, \sigma) \\
& =\left(\frac{\sigma^{2}}{1-\sigma^{4}}\right)[A(t, \sigma)+M(t, \sigma) B(t, \sigma)],
\end{aligned}
$$

where

$$
A(t, \sigma)=y_{00}(t)+\sigma^{2} y_{01}(t)
$$

and $M(t, \sigma)$ and $B(t, \sigma)$ are given in (75). An analogous functional form is obtained for the stable manifold

$$
\begin{aligned}
\tilde{u}_{s}(t-\sigma, \sigma)=\tilde{v}_{s}(t, \sigma)= & \left(\frac{\sigma^{2}}{1-\sigma^{4}}\right)[A(-t, \sigma) \\
& +M(-t, \sigma) B(-t, \sigma)] .
\end{aligned}
$$

The leading term $A(t, \sigma)$ in these approximations is an even function of $t$. Thus the leading terms in the two approximations are identical and equal to $A(t, \sigma)$. This term corresponds to the backbone on which the homoclinic oscillations, defined by the exponential terms, are imposed. It will be called the deformed separatrix.

The second term in (81) is responsible for the splitting of the separatrix. It is the product of two factors. The first factor $M(t, \sigma)$, which is given in (75), is exponentially small in $\sigma$ and results from the matching described in Section III E. In the infinite past and future it has the values $M(-\infty, \sigma)=0$ and $M(\infty, \sigma)=84 \pi \sigma^{-6} \exp \left(-\sqrt{2} \pi^{2} / \sigma\right) K$ where $K$ is the Stokes constant divided by $2 \pi i$. Further, the switching function $S(t)$ is a monotonically increasing function of $t$ and is expected to take the values $S(-\infty)=0, S(0)=1$, and $S(\infty)$ $=2$. This factor is responsible for switching on (off) the homoclinic oscillations in the unstable (stable) manifold.

The second factor $B(t, \sigma)$ gives rise to the homoclinic oscillations. It is an odd oscillatory function of $t$ with period $\sigma$. The amplitudes of the oscillations increase exponentially as $e^{\sqrt{2} t}$ in the limit $t \rightarrow \pm \infty$.

\section{B. The deformed separatrix}

The leading term in the approximation is given by

$$
\begin{aligned}
A(t, \sigma)= & y_{00}(t)+\sigma^{2} y_{01}(t) \\
= & -1+3 \tanh ^{2} \tau+\frac{\sigma^{2}}{8}(2(1-2 \tau \tanh ) \tau \\
& \left.-15 \operatorname{sech}^{2} \tau\right) \operatorname{sech}^{2} \tau,
\end{aligned}
$$

where $\tau=t / \sqrt{2}$. In Fig. 5 the deformed separatrix is compared with the zero-order separatrix of the Hénon map for two values of $\sigma=0.55, \quad 0.75 \quad(\lambda=-0.90849375$, $-0.68359375)$. The dashed line is the solution of the unperturbed problem, i.e., the term $y_{00}(t)$, and the solid line is the deformed separatrix, i.e., the sum of the terms $y_{00}(t)$ $+\sigma^{2} y_{01}(t)$. This approximation is very good in the neigh- 

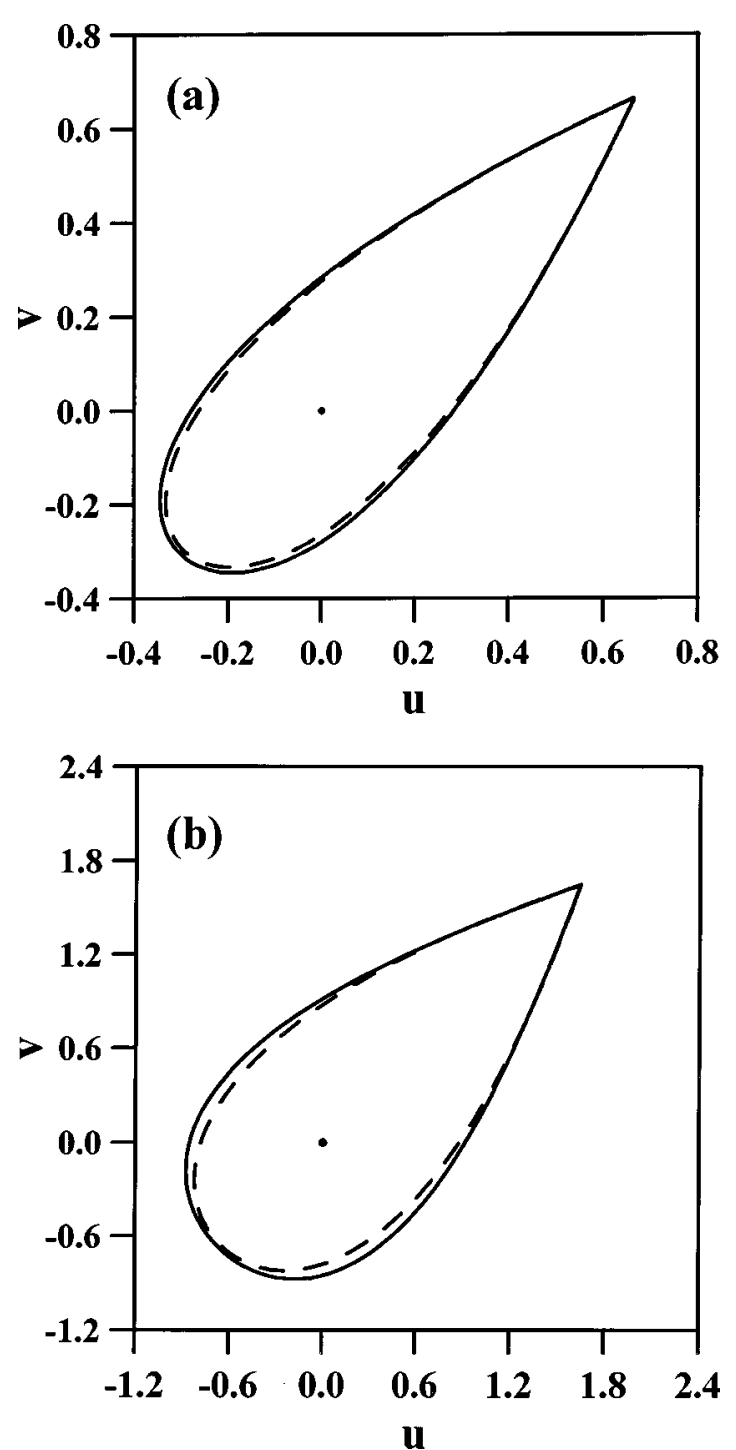

FIG. 5. Comparison of the zero-order separatrix (dashed line) and the firstorder deformed separatrix (solid line) of the Hénon for two values of $\sigma$. (a) $\sigma=0.55$, (b) $\sigma=0.75$. The deviation between the zero-order separatrix and the deformed separatrix is not observable for smaller values of $\sigma$.

borhood of the unstable periodic orbit (note the difference in the scale of the axis in these figures).

\section{The homoclinic oscillations}

The oscillatory factor $B(t, \sigma)$, which is responsible for the homoclinic oscillations, is given by

$$
\begin{aligned}
B(t, \sigma)= & v_{2}(t) \sin \left(\frac{2 \pi t}{\sigma}\right)-\frac{5}{48 \sqrt{2}} v_{1}(t) \cos \left(\frac{2 \pi t}{\sigma}\right) \\
= & {\left[\frac{5}{8}(\tau \tanh \tau-1) \operatorname{sech}^{2} \tau-\frac{5}{24}\right.} \\
& \left.+2 \frac{1}{12} \cosh ^{2} \tau\right] \sin \left(\frac{2 \pi t}{\sigma}\right) \\
& -\left[\frac{5 \pi}{16} \tanh \tau \operatorname{sech}^{2} \tau\right] \cos \left(\frac{2 \pi t}{\sigma}\right),
\end{aligned}
$$

where $\tau=t / \sqrt{2}$. This function is plotted for two different

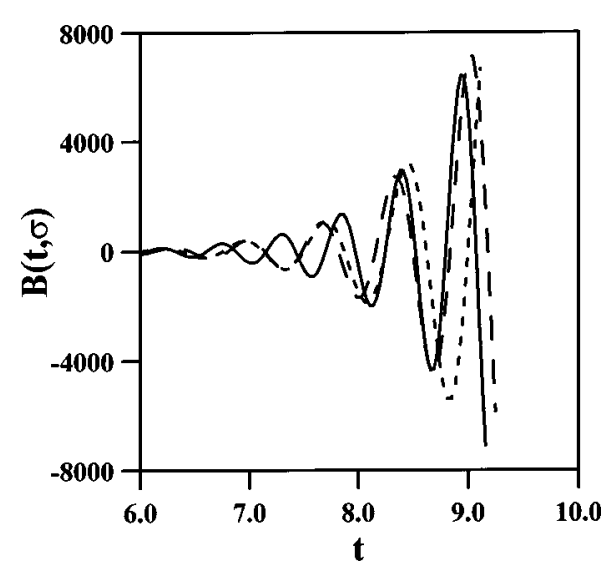

FIG. 6. The $B(t, \sigma)$ as a function of time $t$ for three values of $\sigma$. Solid line $\sigma=0.55$, dashed line $\sigma=0.68$, and dotted line $\sigma=0.75$.

values of $\sigma=0.55, \quad 0.75 \quad(\lambda=-0.90849375$, $-0.68359375)$ in Fig. 6. There are two features that should be observed. First, the amplitudes of the oscillations are not dependent on the parameter $\sigma$, and second, the periods of oscillation are dependent on $\sigma$.

\section{Evaluation of $K$}

According to (55), the constant $K$ is given by

$$
K=\lim _{\substack{p \rightarrow 2 \pi i \\ \operatorname{Im} p<2 \pi i}}(p-2 \pi i) A(p),
$$

where

$$
A(p)=\mathscr{B}\left[z^{-4} \hat{\Phi}_{0}(z)\right](p),
$$

and $\hat{\Phi}_{0}(z)$ is the formal power series solution of the inner equation (41). It follows from the well-known properties of the Laplace transform that

$$
A(p)=D_{p}^{-4} V(p),
$$

where $V(p)$ is the solution of the convolution equation (48) and $D_{p}^{-1}$ is the operator of integration with respect to the variable $p$.

Providing that

$$
V(p)=\sum_{k=1}^{\infty} v_{k} p^{2 k-1}
$$

one finds

$$
A(p)=\sum_{k=1}^{\infty} \frac{v_{k}(2 k-1) !}{(2 k+3) !} p^{2 k+3}=p^{3} B(p),
$$

where $B(p)$ is an even function which possesses the same singularities at the points $p= \pm 2 \pi i$ as $A(p)$. In the leading term, these singularities are first-order poles. So

$$
B(q)=\sum_{k=1}^{\infty} B_{k} q^{2 k} \approx \frac{\chi}{q^{2}+1},
$$

where $p=2 \pi q$ and

$$
B_{k}=\frac{v_{k}(2 k-1) !}{(2 k+3) !}(2 \pi)^{2 k} .
$$




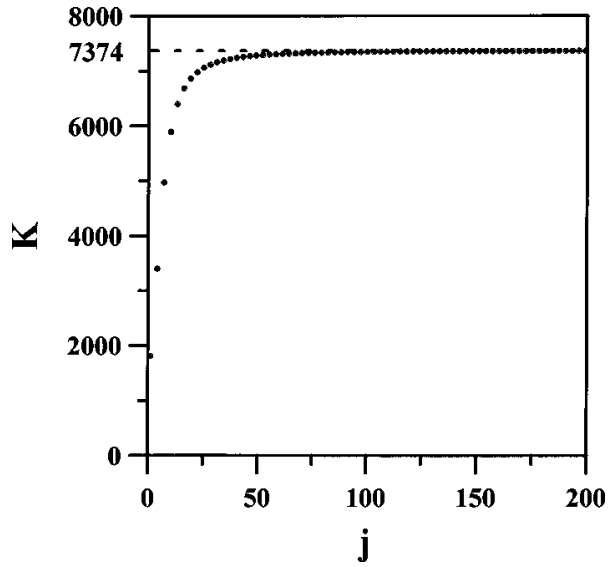

FIG. 7. Values of approximation of the constant $K$ given by (89) versus the number of iterations $j$.

Since

$$
\frac{\chi}{q^{2}+1}=\chi\left(1-q^{2}+q^{4}-\cdots\right)
$$

we obtain

$$
\chi=\lim _{k \rightarrow \infty}(-1)^{k} B_{k} .
$$

So,

$$
\lim _{q \rightarrow i}(q-i) B(q)=\chi \lim _{q \rightarrow i} \frac{q-i}{q^{2}+1}=-\frac{\chi}{2} i
$$

Now,

$$
\begin{aligned}
K=\lim _{q \rightarrow i}(2 \pi q-2 \pi i) A(2 \pi q) & =(2 \pi)^{4} \lim _{q \rightarrow i}(q-i) q^{3} B(q) \\
& =-\frac{(2 \pi)^{4}}{2} \chi .
\end{aligned}
$$

In order to obtain the numerical value of $K$, one needs to find the recurrence equation for $v_{k}$ from the equation (48). Since for any positive $\alpha, \beta$

$$
p^{\alpha *} p^{\beta}=\frac{\Gamma(\alpha+1) \Gamma(\beta+1)}{\Gamma(\alpha+\beta+2)} p^{\alpha+\beta+1},
$$

hence according to (85)

TABLE I. The numerical estimation of $K$.

\begin{tabular}{cccccc}
\hline \hline \multicolumn{2}{c}{$\sigma=0.55$} & \multicolumn{2}{c}{$\sigma=0.60$} & \multicolumn{2}{c}{$\sigma=0.68$} \\
$t$ & $K$ & $t$ & $K$ & $t$ & $K$ \\
\hline 0.01 & 7234.98 & 0.01 & 7222.66 & 0.005 & 7413.17 \\
0.02 & 7204.58 & 0.02 & 7242.71 & 0.010 & 7731.35 \\
0.03 & 7189.23 & 0.03 & 7250.00 & 0.015 & 7838.09 \\
0.04 & 7178.80 & 0.04 & 7257.16 & 0.020 & 7892.68 \\
0.05 & 7173.38 & 0.05 & 7266.78 & 0.015 & 7926.99 \\
\hline \hline
\end{tabular}

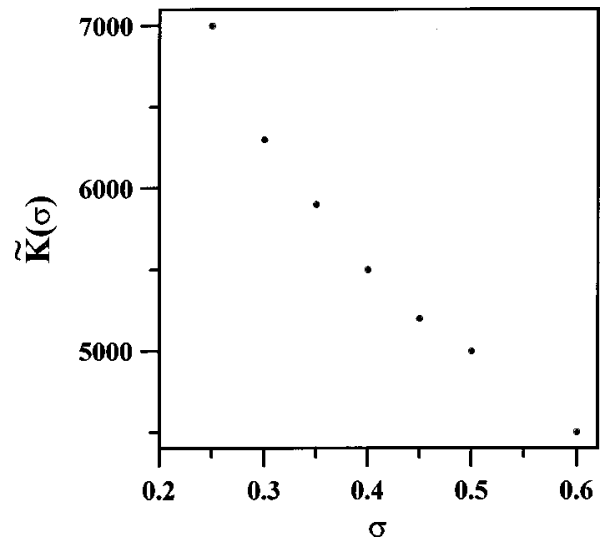

FIG. 8. Values of $\widetilde{K}(\sigma)$ as a function of $\sigma$. These values of $\widetilde{K}(\sigma)$ were obtained by visually fitting the approximate solution with the numerical results (see Fig. 9).

$$
\begin{aligned}
V^{*} V(p)= & \sum_{m=2}^{\infty} \frac{p^{2 m-1}}{(2 m-1) !} \\
& \times \sum_{k+j=m} v_{k} v_{j}(2 k-1) !(2 j-1) !
\end{aligned}
$$

Taking into account the expansion
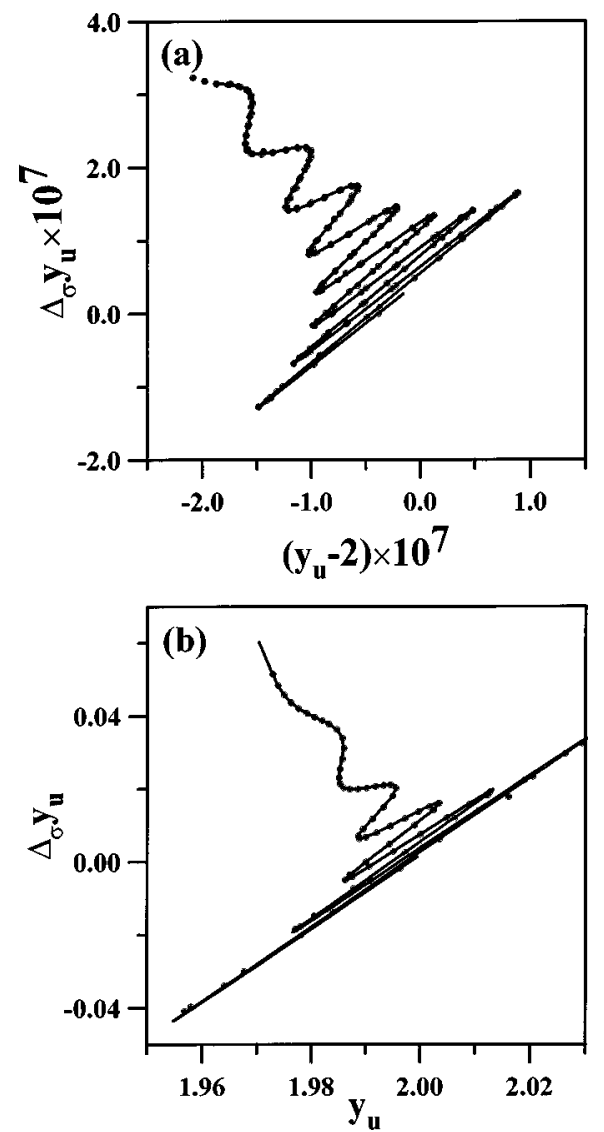

FIG. 9. Comparison of the fit of the approximate unstable manifold (solid line) of the map given in (23) with the numerical result (dots) for two values of $\sigma$, (a) $\sigma=0.25$ and (b) $\sigma=0.50$ in the $\left(y, \Delta_{\sigma} y\right)$ plane. The values of $\widetilde{K}(\sigma)$ shown in Fig. 8 were obtained by fitting these two curves in the vicinity of the first time that $\Delta_{\sigma} y_{u}(t) .=0$. 

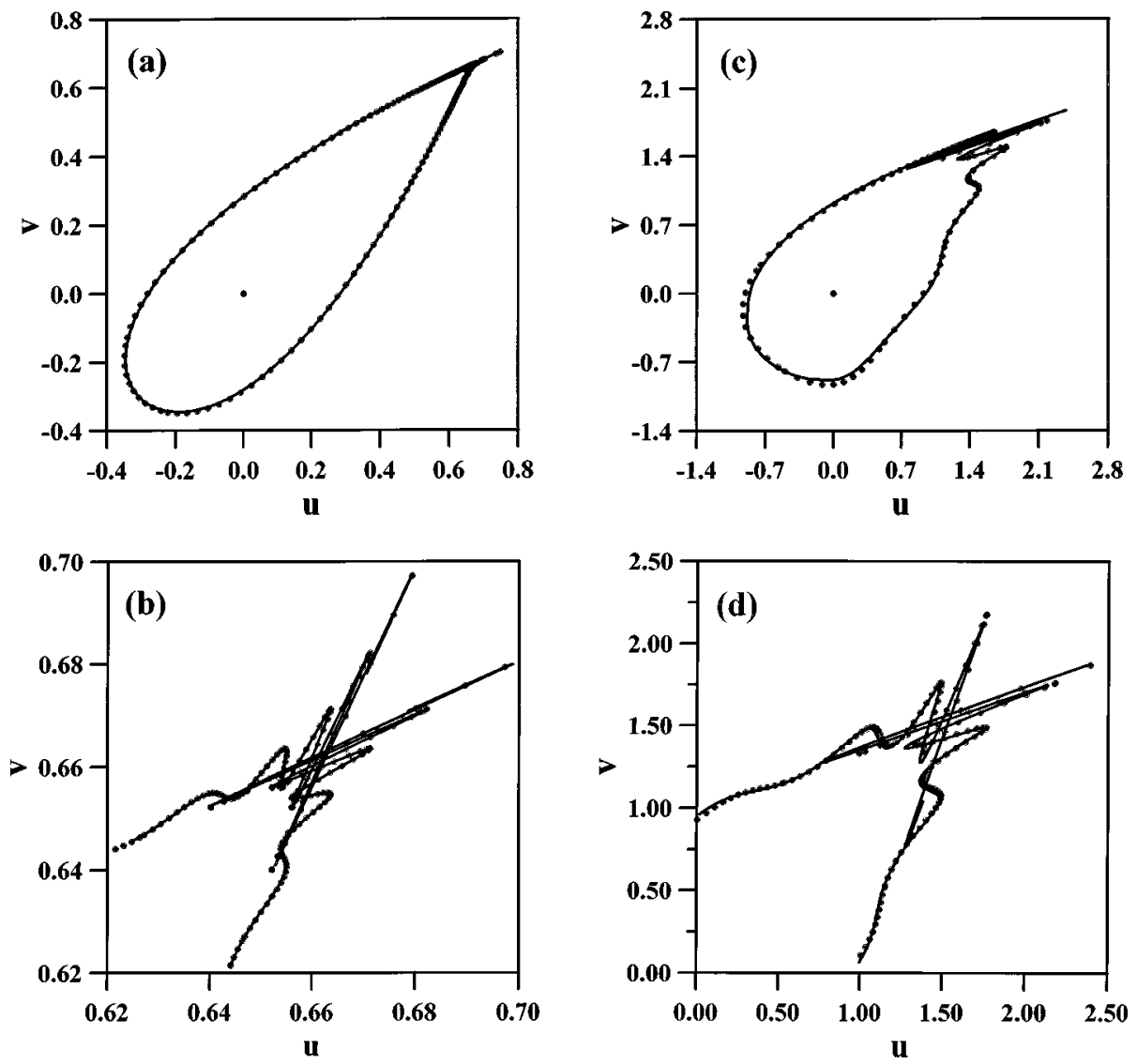

FIG. 10. Comparison of the first-order approximation and numerical results for the Hénon map. In (a) and (c) the first-order approximation of the unstable manifold is compared with the numerically determined manifold for two values of $\sigma$ (a) $\sigma=0.55$, (c) $\sigma=0.75$. In (b) and (d) the first-order approximation of the homoclinic tangle is compared with the numerically determined tangle in the vicinity of the unstable periodic orbit for the same two values of $\sigma$ (b) $\sigma$ $=0.55$, (d) $\sigma=0.75$. The solid line is the analytical approximation and the dots are the points on the numerically determined manifolds. Note that these plots are in the $(u, v)$ phase space.

$$
2(\cosh p-1)=p^{2}+p^{4}\left(\frac{2}{4 !}+\frac{2 p^{2}}{6 !}+\frac{2 p^{4}}{8 !}+\cdots\right),
$$

one finds that the recurrence equation $v_{1}=6$,

$$
\begin{aligned}
& {[1-6 /(2 \ell-1)(\ell-1)] v_{\ell-1}} \\
& =1 /(2 \ell-1) ! \sum_{\substack{k+j=1 \\
k, j>1}} v_{k} v_{j}(2 k-1) !(2 j-1) ! \\
& \quad-2 \sum_{j=2}^{\ell-[1]} v_{l-j} /(2 j) !,
\end{aligned}
$$

when $l>2$, follows from the convolution equation (48) written as

$$
p^{2} V(p)=V^{*} V(p)-2 p^{4}\left(\frac{1}{4 !}+\frac{p^{2}}{6 !}+\cdots\right) V(p) .
$$

So, according to (86)-(89)

$$
K=-\frac{(2 \pi)^{4}}{2} \lim _{j \rightarrow \infty}(-1)^{j} \frac{v_{j}(2 \pi)^{2 j}}{(2 j+3)(2 j+2)(2 j+1) 2 j},
$$

where $v_{j}$ is given by (60).

The numerical evaluation of the Stokes constant using (90) is difficult due to the slow convergence of the recur- rence equation (60). Numerically, the value $K \approx 7374$ was obtained for $j=200$. The values of $K$ versus $j$ are plotted in Fig. 7. (As the authors have learned from one of the referees, the obtained numerical value of $K$ is in agreement with the numerical value $K \approx 7364.36188$ in Ref. 42 .)

In order to confirm this value of the Stokes constant we considered the distance $d=\left|y_{s}(t, \sigma)-y_{u}(t, \sigma)\right|$ between the stable and unstable solutions of the perturbed equation (15) in the vicinity of $t=0$. Here the value of the switching function is approximately equal to unity $S(t \approx 0, \sigma) \approx 1$ and consequently we obtain the following estimate for the Stokes constant

$$
K=\frac{\sigma^{6} d}{84 \pi} e^{\sqrt{2} \pi^{2} / \sigma} B^{-1}(t, \sigma) .
$$

Numerical estimates of $K$, based on numerically evaluated $d$, are given in Table I. They are consistent with the value $K$ obtained above.

\section{E. The switching function}

Our purpose is approximation of the unstable manifold $W_{u}$ in the region of homoclinic oscillations. For this $S(t, \sigma)$ can be approximated by 


$$
\widetilde{S}(t)= \begin{cases}0, & t<0 \\ 1, & t=0 \\ 2, & t>0 .\end{cases}
$$

This approximation is based on the assumption that for small $\sigma$ the switching function turns on rapidly in a neighborhood $t=0$. It works well for sufficiently small $\sigma$ (for example, for $\sigma=0.25$ ) however, as $\sigma$ increases (in the range between 0.3 and 0.7), we observe increasing deviation of $S(t, \sigma)$ from $\widetilde{S}(t)$ in the region where the homoclinic oscillations become large. In other words, for larger $\sigma$ the best fit between numerical simulation and analytic approximation (78) of the unstable manifold in the region of homoclinic oscillations occurs when $S(t, \sigma)$ is noticeably smaller than 2 . This deviation cannot be attributed to the less rapid turn on of the switching function $S(t, \sigma)$ as $\sigma$ gets larger, since approximately the same value of $S(t, \sigma)$ is valid for all $t$ in the region of our approximation.

For the purpose of convenience we choose to retain the approximation $\widetilde{S}(t)$ for $S(t, \sigma)$, replacing instead the constant $K$ in (75)-(78) by what we call the constant of the best fit $\widetilde{K}(\sigma)$. Experimental dependence of $\widetilde{K}(\sigma)$ on $\sigma$ is shown in Fig. 8. These values of $\widetilde{K}(\sigma)$ were determined by fitting the analytical approximation of the unstable manifold of (16) with the numerical results. Two examples are shown in Fig. 9. Notice that $\widetilde{K}(0.25)$ is "close" to the "theoretical value", $K=7374$. The nature of the dependence $\widetilde{K}(\sigma)$ on $\sigma$ is expected to become clearer through the further study of the problem. Finally, based on Table I and Fig. 8 we observe that $S(t, \sigma)$ deviates from $\widetilde{S}(t)$ faster in the region of homoclinic oscillations than at $t \approx 0$ (near the primary intersection point) as $\sigma$ increases. As far as we know, most numerical studies of the splitting phenomenon have been in the vicinity of the primary intersection point. This can, probably, explain why the dependence $\widetilde{K}(\sigma)$ was not observed numerically in the literature, though asymptotic expansion of the pre-exponential factor in powers of $\sigma$ for the standard map was conjectured and discussed in Ref. 24.

\section{F. Homoclinic tangle}

Our numerical data is based on the iterations of the map $T$ given by (23). A point $P_{0}$ is taken on the unstable manifold $W_{u}$ of $(23)$ in a close proximity $\left(\sim 10^{-13}\right)$ of the hyperbolic fixed point $(2,0)$ of $T$. Then the segment $\left[P_{0} P_{1}\right]$, where $P_{1}=T P_{0}$, lies very close to $W_{u}$. This segment is divided into a number of points $(\sim 30)$. Iterations of these points numerically define the unstable manifold $W_{u}$. Numerical construction of the stable manifold $W_{s}$ is based on time reversibility of the map $T$.

The analytical approximation of the stable and unstable manifolds of the Hénon map are compared to the numerically determined manifolds in Figs. 10(a) and 10(c) for two values of $\sigma=0.55, \quad 0.75 \quad(\lambda=-0.90849375$, $-0.68359375)$. The homoclinic tangle in the vicinity of the unstable periodic orbit constructed from the analytical approximation is compared with the numerical results in Figs. $10(\mathrm{~b})$ and $10(\mathrm{~d})$ for the same values of $\sigma$. In these figures the
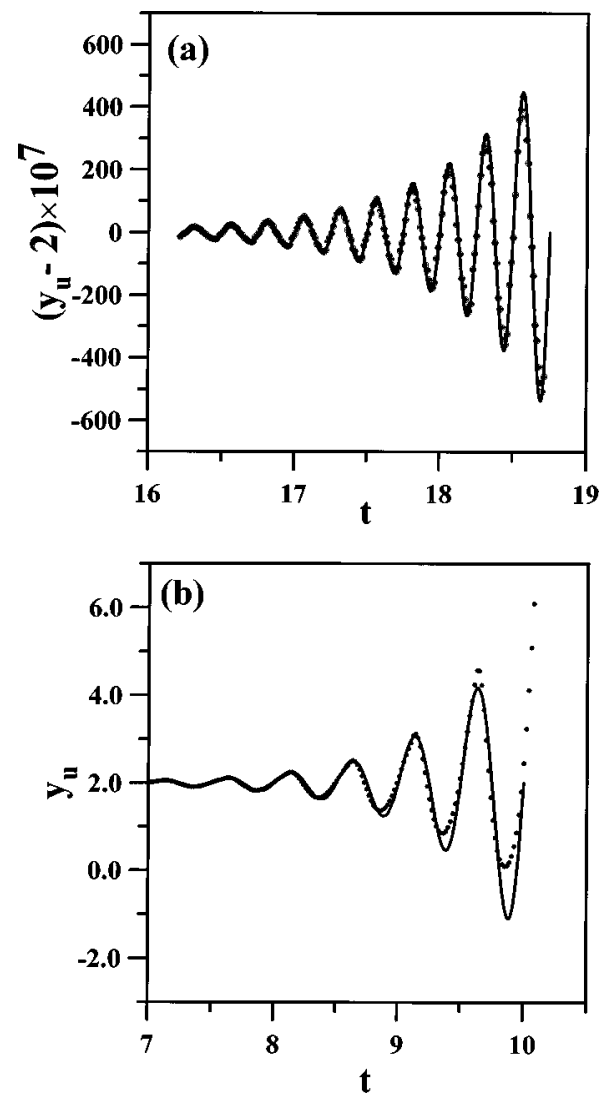

FIG. 11. Comparison of the approximate solution, $\tilde{y}_{u}(t, \sigma)$ (solid line) with the numerical solution (dots) as a function of time for two value of $\sigma$, (a) $\sigma=0.25$, (b) $\sigma=0.50$. Here we see that the approximation is excellent for times significantly longer than can be observed in the phase plane plots seen in Figs. 9 and 10.

solid line is the analytical approximation and the dots are points on the numerically determined manifolds. The agreement between the analytical approximation and the numerically determined manifolds is excellent for $\sigma=0.55$. As the value of $\sigma$ is increased, it is observed that the agreement between the approximate solution and the numerical results is not as good far from the unstable periodic orbit. This discrepancy is attributed to the approximation of the deformed separatrix (see Fig. 5) since the last term in (78) is negligible in this region. The agreement between the approximate solutions and the numerical solutions in the vicinity of the unstable periodic orbit is excellent for all values of $\sigma$ considered. In Fig. 11 we compare the approximate solution $\tilde{y}_{u}(t, \sigma)$ in the $(y, t)$ plane with the corresponding numerical values for two values of $\sigma$, (a) $\sigma=0.25$ and (b) $\sigma=0.50$. Here we see that $\tilde{y}_{u}(t, \sigma)$ approximates the homoclinic oscillations at nearly two times longer in time $t$ than we are able to observe in the phase plane; final times in Figs. 9(a) and 9 (b) are $t=14$ and $t=6$, respectively. Moreover, we observe that the approximation starts to fail first at the bottoms of the oscillations, which correspond to the lobes inside the pseudoseparatrix. We attribute this to the fact that the inside lobes start to bend into a horseshoe-type shape. It is expected that higher-order approximations will shed some light in this phenomenon. 


\section{ACKNOWLEDGMENTS}

This research is supported by the National Science Foundation (Grant No. DMS 96500644 and RII-8922106).

${ }^{1}$ A. Delshams and R. Ramirez-Ros, "Poincaré-Melnikov-Arnold methods for analytic planar maps,' Nonlinearity 9, 1-26 (1996).

${ }^{2}$ P. Holmes, J. Marsden, and J. Scheurle, 'Exponentially small splitting of separatrices with application to KAM theory and degenerate bifurcations," Contemp. Math. 81, 213-244 (1988).

${ }^{3}$ M. D. Kruskal and H. Degur, "Asymptotics beyond all orders in a model of crystal growth,' Stud. Appl. Math. 85, 129-181 (1991).

${ }^{4}$ V. F. Lazutkin, "Splitting of separatrices for the Chirikov's standard map,' Preprint VINITI 6372/84, 1984 (Russian).

${ }^{5}$ B. Fiedler and J. Scheurle, "Discretization of homoclinic orbits, rapid forcing and 'invisible' chaos,' Mem. Am. Math. Soc. 119 (1996), no. 570.

${ }^{6}$ W. P. Reinhardt, 'Regular and irregular correspondence-Adiabatic invariants in classical and quantum mechanics," Prog. Theor. Phys. Suppl. 116, 179-205 (1994); S. Bleher and W. P. Reinhardt, 'Quantum-classical correspondence for classically chaotic dynamics,' Comments At. Mol. Phys. 25, 133-149 (1990); W. P. Reinhardt, "Chaos and collisions: Introductory concepts,' in Collision Theory for Atoms and Molecules, edited by F. A. Gianturco (Plenum, New York, 1989).

${ }^{7}$ M. J. Davis and S. K. Gray, "Unimolecular reactions and phase space bottlenecks,', J. Chem. Phys. 84, 5389-5411 (1986).

${ }^{8}$ Asymptotics Beyond All Orders, Vol. 284 of NATO Asi Series, Series B, Physics, edited by H. Segur, S. Tanveer, and H. Levine (Plenum, New York, 1992).

${ }^{9}$ A. Tovbis, "Asymptotics beyond all orders and analytic properties of inverse Laplace transforms of solutions,' Commun. Math. Phys. 163, 245-255 (1994).

10 C. J. Amick and J. B. McLeod, "A singular perturbation problem in needle crystals,'” Arch. Ration. Mech. Anal. 109, N2, 139-171 (1992).

11 J. M. Hammersley and G. Mazzarino, "A differential equation connected with the dendritic growth of crystals," IMA J. Appl. Math. 42, 43-75 (1989).

${ }^{12}$ C. J. Amick and J. B. McLeod, "A singular perturbation problem inwater waves,' Stab. Appl. Anal. Cont. Media, 127-148 (1992).

${ }^{13}$ W. Eckhaus, "Singular perturbations of homoclinic orbits in $R^{4}$," SIAM (Soc. Ind. Appl. Math.) J. Math. Anal. 23, 1269-1290 (1992).

14 R. Grimshaw, "The use of Borel-summation in the establishment of nonexistence of certain travelling-wave solutions of the KuramotoSivashinsky equation,', Wave Motion 15, 393-395 (1992).

15 Y. Pomeau, A. Ramani, and B. Grammaticos, "Structural stability of the Korteweg-de Vries solutions under a singular perturbation, Physica D 31, 127-134 (1988).

16 R. Grimshaw and N. Joshi, "Weakly non-local solitary waves in singularly perturbed Korteweg-de Vries equation,', SIAM (Soc. Ind. Appl. Math.) J. Appl. Math. 55, 124-135 (1995).

17 V. Hakkim and K. Mallick, "Exponentially small splitting of separatrices, matching in the complex plane and Borel summation,' Nonlinearity 6, 57-70 (1993).

18 C. Amick, E. S. C. Ching, L. P. Kanadoff, and V. Rom-Kedar, "Beyond all orders: singular perturbation of a mapping,' J. Nonlinear Sci. 2, 6-67 (1992).

${ }^{19}$ A. Tovbis, "On exponentially small terms of solutions to nonlinear ordi- nary differential equations,', Methods Applications Anal. 1, 57-74 (1994).

${ }^{20}$ B. M. Herbst and M. J. Ablowitz, "Numerical chaos, symplectic instabilities, and exponentially small splitting distances,' J. Comput. Phys. 105, 122-132 (1993).

21 V. F. Lazutkin, I. G. Schachmannski, and M. B. Tabanov, "Spitting of separatrices for standard and semistandard mappings," Physica D 40, 235-248 (1988)

${ }^{22}$ E. Fontich and C. Simó, "The splitting of separatrices for analytic diffeomorphisms,' Ergodic Theory Dynamical Syst. 10, N2, 295-318 (1990).

${ }^{23}$ E. Fontich and C. Simó, "Invariant manifolds for near identity differentiable maps and splitting of separatrices,' Ergodic Theory Dynamical Syst. 10, N2, 319-346 (1990).

${ }^{24}$ V. G. Gelfreich, V. F. Lazutkin, and N. V. Svanidze, "A refined formula for the separatrix splitting for the standard map,' Physica D 74, 82-101 (1994).

${ }^{25}$ A. Tovbis, M. Tsuchiya, and C. Jaffé, “Exponential asymptotic expansions and approximations of the unstable and stable manifolds of the Hénon map," preprint, 1994.

${ }^{26}$ A. Tovbis, M. Tsuchiya, and C. Jaffé, "Chaos-integrability transition in nonlinear dynamical systems: exponential asymptotic approach," Differential Equations and Applications to Biology and to Industry, edited by M. Martelli, K. Cooke, E. Cumberbatch, B. Tang, and H. Thieme (World Scientific, Singapore, 1996), pp. 495-507.

${ }^{27}$ K. Nakamura and M. Hamada, "Asymptotic expansion of homoclinic structures in a symplectic mapping,' J. Phys. A 29, 7315-7327 (1996).

${ }^{28}$ K. Nakamura, Quantum versus Chaos: Questions Emerging from Mesoscopic Comos (Kluwer Academic, Boston, 1997).

${ }^{29}$ K. Chandrasekharan, Elliptic Functions (Springer-Verlag, New York, 1985).

${ }^{30}$ M. Hénon, "Numerical study of quadratic area-preserving mapping,', Q. Appl. Math. 27, 291-312 (1969).

${ }^{31}$ A. O. Gelfond, Calculus of Finite Differences (Nauka, Moscow, 1967) (in Russian).

${ }^{32}$ D. K. Arrowsmith and C. M. Place, An Introduction to Dynamical Systems (Cambridge University Press, Cambridge, England, 1990).

${ }^{33}$ V. Lazutkin, 'Splitting of complex separatrices,', Funct. Anal. Appl. 22, N2, 154-156 (1988).

${ }^{34} \mathrm{~V}$. Arnold and Yu. Il'yashenko, Ordinary Differential Equations, Dynamical Systems 1 (Springer-Verlag, New York, 1988).

${ }^{35}$ A. Fruchard and J. Schaefke, "Exponentially small splitting of separatrices for differential equations with small step size,' J. Dynamical Control Syst. 2, 193-238 (1996).

${ }^{36}$ G. Immink, “Asymptotics of analytic difference equations,', Lect. Notes Math., 1085 (1984).

${ }^{37}$ W. Wasow, Asymptotic Expansions for Ordinary Differential Equations (Dover, New York, 1976).

${ }^{38}$ J. Martinet and J. P. Ramis, "Elementary acceleration and multisummability,' Ann. Inst. Henri Poincaré Phys. Theor. 54, 1-71 (1991).

${ }^{39}$ A. Tovbis, 'Laplace transformations of nonlinear ODE's and connection problems," (in preparation).

${ }^{40}$ M. V. Berry, "Uniform asymptotic smoothing of Stokes's discontinuities,', Proc. R. Soc. London, Ser. A 422, 7-21 (1989).

41 J. B. McLeod, "Smoothing of Stokes discontinuities, Proc. R. Soc. London, Ser. A 437, 343-354 (1992).

${ }^{42}$ V. Chernov, "Separatrix splitting for the Hénon map: a resurgent approach,' University of Barcelona, Math. Preprint Series 188, 1995. 